\title{
Isolated Horizons: Hamiltonian Evolution and the First Law
}

\author{
Abhay Ashtekar ${ }^{1,2} *$, Stephen Fairhurst ${ }^{1 \dagger}$ and Badri Krishnan ${ }^{1 \ddagger}$ \\ 1. Center for Gravitational Physics and Geometry \\ Department of Physics, The Pennsylvania State University \\ University Park, PA 16802, USA \\ 2. Institute for Theoretical Physics, \\ University of California, Santa Barbara, CA 93106, USA
}

\begin{abstract}
A framework was recently introduced to generalize black hole mechanics by replacing stationary event horizons with isolated horizons. That framework is significantly extended. The extension is non-trivial in that not only do the boundary conditions now allow the horizon to be distorted and rotating, but also the subsequent analysis is based on several new ingredients. Specifically, although the overall strategy is closely related to that in the previous work, the dynamical variables, the action principle and the Hamiltonian framework are all quite different. More importantly, in the non-rotating case, the first law is shown to arise as a necessary and sufficient condition for the existence of a consistent Hamiltonian evolution. Somewhat surprisingly, this consistency condition in turn leads to new predictions even for static black holes. To complement the previous work, the entire discussion is presented in terms of tetrads and associated (real) Lorentz connections.
\end{abstract}

\section{INTRODUCTION}

The zeroth and first laws of black hole mechanics refer to equilibrium situations and small departures therefrom. The standard treatments [1 5] restrict themselves to stationary

\footnotetext{
*E-mail: ashtekar@gravity.phys.psu.edu

${ }^{\dagger}$ E-mail: fairhurs@phys.psu.edu

${ }_{\ddagger}^{\ddagger}$ E-mail: krishnan@phys.psu.edu
} 
space-times admitting event horizons and small perturbations from stationarity. While this simple idealization is a natural starting point, from physical considerations it seems overly restrictive. (See [6,7] and especially [8] for a detailed, critical discussion.) A framework, which is tailored to more realistic physical situations was introduced in [6] and the zeroth and first laws were extended to it in $[7-9]$. This analysis generalizes black hole mechanics in two directions. First, the notion of event horizons is replaced by that of 'isolated horizons'. While the former can only be defined retroactively, requiring access to the entire space-time history, the latter can be defined quasi-locally. Second, the underlying space-time need not admit any Killing field; isolated horizons need not be Killing horizons. The static event horizons normally used in black hole mechanics [1-3, 10] and the cosmological horizons in de Sitter space-times [11] are all special cases of isolated horizons. Furthermore, since spacetimes can now admit gravitational and matter radiation, there is a large class of other examples.

The framework developed in [8] for generalizing black hole mechanics was based on two restrictive assumptions. First, only undistorted, non-rotating horizons were considered. That is, the boundary conditions used in [8] implied that the intrinsic 2-metric of the horizon is spherically symmetric and that the imaginary part of the Weyl tensor component $\Psi_{2}$ which encodes the angular momentum — vanishes. Second, while a rather general class of matter fields was allowed, it was assumed that the only relevant charges - i.e., hair — are the standard electromagnetic ones. The second assumption was weakened in [9, 12] which allowed dilaton couplings and Yang-Mills fields. In this paper, we allow for distortion and more general matter sources. Distortion plays an important role in several astrophysical situations, e.g., in problems involving black holes immersed in external fields, or surrounded by matter rings, and especially in the problem of black hole collisions. Post-Newtonian considerations suggest that, during black hole coalescence, individual horizons are distorted due to the Coulomb attraction even in the regime in which the black holes are sufficiently far from each other for the gravitational radiation falling into their horizons to be negligible. This phenomenon is also seen in numerical simulations.

The extensions [9,12] which incorporated dilatonic and Yang-Mills charges did not involve a significant generalization of the basic framework developed in [8]. The present paper, on the other hand, does. We begin with substantially weaker boundary conditions (formulated in terms of (real) tensor fields rather than the spinors used in [8]), and show that they imply constancy of surface gravity (and electro-static potential) on the horizon. This property turns out to be necessary and sufficient for the usual action principle of tetrad gravity to continue to be valid in presence of isolated horizons. The action leads to a covariant phase space, constructed from solutions to the field equations. Ref [8], by contrast, used the canonical phase space based on spinorial variables which is tailored for quantization but which contains

\footnotetext{
${ }^{1}$ However, it allowed space-times, such as the Robinson-Trautman solutions, in which there is no space-time Killing field whatsoever in any neighborhood of the isolated horizon [15].
} 
technical complications that are unnecessary to the classical mechanics of isolated horizons. Up to this point, distortion and rotation are both incorporated. However, in the last step, i.e., in the discussion of the first law, we restrict ourselves to non-rotating horizons. (Rotation is incorporated in [13].)

To formulate the first law one must first define the energy $E_{\Delta}$ associated with any isolated horizon $\Delta$. Since there can be radiation in the spacetime outside isolated horizons, the ADM energy $E_{\mathrm{ADM}}$ is not a good measure of $E_{\Delta}$ [0,8]. Instead, as in the work of Brown and York [14, the strategy is to define the energy of the horizon using a Hamiltonian framework. Experience with the phase space formulation of general relativity suggests that, in the presence of boundaries, the Hamiltonian $H_{t}$ generating time-translation along a suitable vector field $t^{a}$ acquires surface terms. The idea is to define $E_{\Delta}$ as the surface term at $\Delta$ in the Hamiltonian. The key issue then is that of selecting the 'appropriate' time-translation $t^{a}$. Since one expects the volume term in the expression of $H_{t}$ to be a linear combination of constraints and thus vanish when evaluated on solutions, the problem reduces to that of specifying the boundary values of $t^{a}$ (or, equivalently, of the lapse and shift fields). The conditions at infinity are obvious and, in any case, will not affect the surface term at $\Delta$. Thus, we need to focus only on the boundary value of $t^{a}$ on $\Delta$.

In the non-rotating case it is clear that, at the horizon, $t^{a}$ should be proportional to the null normal to $\Delta$. However, our boundary conditions do not select the null normal uniquely; there is a freedom to rescale the normal by a constant (on $\Delta$ ) which can vary from one space-time to another. This freedom is physically important because, amongst other things, the surface gravity is sensitive to it. Suppose we fix this freedom by tying the boundary value of $t^{a}$ to fields on the horizon, e.g., by demanding that surface gravity be a specific function of the area and charges. We can then ask whether the time-evolution generated by this $t^{a}$ preserves the symplectic structure. It turns out that the answer is not always in the affirmative. On the horizon, the evolution vector field $t^{a}$ and the electromagnetic potential $A_{a}$ have to be tied to the horizon parameters appropriately. These conditions impose a constraint on the surface gravity $\kappa_{(t)}$ and the electric potential $\Phi_{(t)}$. Somewhat surprisingly, the constraint is precisely the first law $\delta E_{\Delta}^{t}=\left(\kappa_{(t)} / 8 \pi G\right) \delta a_{\Delta}+\Phi_{(t)} \delta Q_{\Delta}$. Thus the evolution defined by $t^{a}$ is Hamiltonian if and only if the first law holds. In this sense, the first law is even more fundamental than it is generally taken to be. Conceptually, this is perhaps the

\footnotetext{
${ }^{2}$ This is not surprising since this freedom exists already on Killing horizons. If the space-time is asymptotically flat and admits a static Killing field globally, one can eliminate this freedom by restricting oneself to that Killing field which is unit at infinity. However, this strategy is not available if there is radiation in the exterior region, or, as in the static solutions representing distorted black holes, the metric fails to be asymptotically flat.

${ }^{3}$ In the undistorted context, while this role of the first law was known to the authors of [8], its importance was not fully appreciated. The importance was noticed independently in [16] and used effectively in [12] to extract physical information on spherical black holes with Yang-Mills hair.
} 
most striking feature of the present framework.

The requirement that the first law hold is not sufficient to fix $t^{a}$ uniquely. Although every $t^{a}$ must be a null normal to $\Delta$, the rescaling of $t^{a}$ from one spacetime to another can depend on the horizon parameters and the first law does not fully determine this parameter dependence. There is an infinite family of parameter-dependent vector fields $t^{a}$ each defining a consistent Hamiltonian evolution and a horizon energy $E_{\Delta}^{t}$. By contrast, at infinity all these vector fields must tend to a time-translation of an universal flat metric, used in the construction of the phase space. Hence, there is only the familiar 3-parameter freedom in the definition of $E_{\mathrm{ADM}}^{t}$, associated with the choice of a rest-frame. Furthermore, in any one space-time, we can eliminate it by simply going to the rest frame and thus extract the total mass $M_{\mathrm{ADM}}$ of the system. Although not necessary for mechanics, it is natural to ask if one can define a similar notion of mass of isolated horizons. The answer is in the affirmative in the Einstein-Maxwell theory. Let us require that $t^{a}$ should not only lead to a consistent Hamiltonian evolution but agree, on static solutions, with the static Killing field which is unit at infinity. Then the horizon value of $t^{a}$ is uniquely determined for all space-times in the phase-space. There is a preferred notion of time-translation, say $t_{o}^{a}$. We can set $M_{\Delta}=E_{\Delta}^{t_{o}}$ and regard $M_{\Delta}$ as the mass of an isolated horizon $\Delta$. In the earlier work on non-distorted horizons [7 9:12], the discussion of the first law was carried out only in the context of these preferred evolution vector fields $t_{o}^{a}$. That derivation is more closely tied to the traditional discussion of the laws in the static context but is not necessary from the more general perspective of isolated horizons. Nonetheless, the availability of a canonical definition of the mass $M_{\Delta}$ is useful for other applications of this framework, e.g. to numerical relativity.

The paper is organized as follows. In Section [1], we specify the boundary conditions defining general isolated horizons, allowing both distortion and rotation. We explain the role of these conditions, compare them with those used in [6] 9, 12] and work out their consequences, including the zeroth law. In Section [II] we introduce the Lagrangian framework based on tetrads and (real) Lorentz connections and in Section IV, the covariant phase space. The first law is discussed in Section $\mathrm{V}$. Upto this point, the focus is on the Einstein-Maxwell theory (although incorporation of the dilaton is straightforward). In Section $\nabla \mathbb{1}$ we extend the framework to incorporate Yang-Mills fields. The horizon mass is introduced in Section VII and subtleties associated with the dilaton and Yang-Mills fields are discussed. For the convenience of readers who may not be familiar with distorted black holes, Appendix A presents a variety of examples and, for convenience of readers who work in the Newman-Penrose formalism, Appendix B summarizes the structure of isolated horizons in that framework.

We have attempted to make this paper self-contained in terms of methodology and technical details. However, the motivation behind isolated horizons and certain properties of our Hamiltonian are the same as those discussed in detail in 8 . Since the inclusion of distortion does not add anything substantial to these issues, we have refrained from repeating that discussion in this paper. 


\section{STRUCTURE OF ISOLATED HORIZONS AND THE ZEROTH LAW}

In this section, we will introduce the basic definitions of isolated horizons and analyze their immediate consequences. The definitions will become progressively stronger. However, even the strongest boundary conditions are significantly weaker than requiring the horizon to be a Killing horizon for a local Killing vector field. By proceeding in steps, we will be able to keep track of the precise assumptions that are needed to obtain various results. Also, the availability of a hierarchy of definitions will be useful in other applications - such as numerical relativity and quantum gravity — which lie beyond the scope of the present paper.

Let us begin by introducing some notation. Throughout this paper we assume that all manifolds and fields under consideration are smooth. Let $\mathcal{M}$ be a 4 -manifold equipped with a metric $g_{a b}$ of signature $(-,+,+,+)$. Let $\Delta$ be a null hypersurface of $\left(\mathcal{M}, g_{a b}\right)$. A future directed null normal to $\Delta$ will be denoted by $\ell$. (In this paper, the term 'null normal' will always refer to a future directed null normal.) Let $q_{a b} \widehat{=} g_{a b}$ be the degenerate intrinsic metric on $\Delta$.f A tensor $q^{a b}$ on $\Delta$ will be called an 'inverse' of $q_{a b}$ if it satisfies $q^{a b} q_{a c} q_{b d} \widehat{=} q_{c d}$. Thus $q^{a b}$ is unique only up to addition of terms of the form $\ell^{(a} V^{b)}$ for some vector field $V$ tangential to $\Delta$. The expansion $\theta_{(\ell)}$ of a specific null normal $\ell$ is defined by $\theta_{(\ell)}=q^{a b} \nabla_{a} \ell_{b}$, where $\nabla_{a}$ is the derivative operator compatible with $g_{a b}$. It is straightforward to check that $\theta_{(\ell)}$ is independent of the choice of $q^{a b}$. With this structure at hand, we can now introduce our first definition.

\section{A. Non-expanding Horizons}

Definition 1: A 3-dimensional sub-manifold $\Delta$ of a space-time $\left(\mathcal{M}, g_{a b}\right)$ is said to be a non-expanding horizon if it satisfies the following conditions:

- (i) $\Delta$ is topologically $S^{2} \times \mathbb{R}$ and null;

- (ii) The expansion $\theta_{(\ell)}$ of $\ell$ vanishes on $\Delta$ for any null normal $\ell$;

- (iii) All equations of motion hold at $\Delta$ and the stress-energy tensor $T_{a b}$ of matter fields at $\Delta$ is such that $-T_{b}^{a} \ell^{b}$ is future directed and causal for any future directed null normal $\ell$.

Note that if conditions (ii) and (iii) hold for one null normal $\ell$ they hold for all.

The role of these conditions is as follows. The restriction on topology is geared to the structure of horizons that result from gravitational collapse. However, it can be weakened.

\footnotetext{
${ }^{4}$ Equalities which hold only at $\Delta$ will be denoted by ' $=$ ' and the pullback of a covariant index will be denoted by an arrow under that index, e.g. $\omega_{a}$ will denote the pullback of the 1-form $\omega_{a}$ to $\Delta$.
} 
One can retain the requirement that the horizon have compact cross-sections but replace $S^{2}$ by a manifold with higher genus. Our main analysis will extend to this case in a straightforward manner. More generally, we can allow $\Delta$ to have non-compact cross-sections, as for example in the case of certain acceleration horizons. The results presented in this section, including our derivation of the zeroth law will go through. However, since such horizons extend to infinity, our Hamiltonian framework will have to be modified appropriately. Finally, one could envisage incorporation of NUT charge. This extension would be even more subtle because, if all fields are smooth, $\Delta$ would be topologically $S^{3}$ and $\ell$ would provide a Hopf fibration. In this case, $\Delta$ would not admit any cross-sections which are everywhere transverse to $\ell$. This extension will be discussed elsewhere.

Requirement (iii) is analogous to the dynamical conditions one imposes at infinity. While at infinity one requires that the metric (and other fields) approach a specific solution to the field equations (the 'classical vacuum'), at the horizon we only ask that the field equations be satisfied. The energy condition involved is very weak; it is implied by the (much stronger) dominant energy condition that is typically used. Thus, the first and the last conditions are quite tame.

The key condition is (ii). It implies, in particular, that the horizon area is constant 'in time' and thus incorporates the idea that the horizon is isolated without having to assume the existence of a Killing field. We will denote the area by $a_{\Delta}$ and refer to $R_{\Delta}$ defined by $a_{\Delta}=4 \pi R_{\Delta}^{2}$ as the horizon radius. All these conditions are satisfied on any Killing horizon (with 2-sphere sections) if gravity is coupled to physically reasonable matter (including perfect fluids, Klein-Gordon fields, Maxwell fields possibly with dilatonic coupling, Yang-Mills fields).

Although the conditions in the definition are quite weak, they have surprisingly rich consequences. We will now discuss them in detail. In some of this analysis it is convenient to use a null-tetrad and the associated Newman-Penrose quantities (see Appendix B and references therein). Given a specific null normal field $\ell^{a}$ to $\Delta$, we can introduce a complex null vector field $m^{a}$ tangential to $\Delta$ and a real, future directed null field $n^{a}$ transverse to $\Delta$ so that the following relations hold: $n \cdot \ell=-1, m \cdot \bar{m}=1$ and all other scalar products vanish. The quadruplet $(\ell, n, m, \bar{m})$ constitutes a null-tetrad. There is of course an infinite number of null tetrads compatible with a given $\ell$, related to one another by restricted Lorentz rotations. Our conclusions will not be sensitive to this gauge-freedom.

(a) Properties of $\ell$ : Since $\ell^{a}$ is a null normal to $\Delta$, it is automatically twist free and geodesic. We will denote the acceleration of $\ell^{a}$ by $\kappa_{(\ell)}$

$$
\ell^{a} \nabla_{a} \ell^{b} \widehat{=} \kappa_{(\ell)} \ell^{b}
$$

Note that the acceleration is a property not of the horizon $\Delta$ itself, but of a specific null normal to it: if we replace $\ell$ by $\ell^{\prime}=f \ell$, then the acceleration changes via $\kappa_{\left(\ell^{\prime}\right)}=f \kappa_{(\ell)}+\mathcal{L}_{\ell} f$.

Since the twist of $\ell$ vanishes, the Raychaudhuri equation implies:

$$
\mathcal{L}_{\ell} \theta_{(\ell)} \widehat{=} \kappa_{(\ell)} \theta_{(\ell)}-\frac{1}{2} \theta_{(\ell)}^{2}-\sigma \bar{\sigma}-R_{a b} \ell^{a} \ell^{b}
$$


where $\sigma=m^{a} m^{b} \nabla_{a} \ell_{b}$ is the shear of $\ell$ in the given null tetrad. Since $\theta_{(\ell)}$ vanishes on $\Delta$, we conclude: $\sigma \bar{\sigma}+R_{a b} \ell^{a} \ell^{b} \hat{=} 0$. The condition on the stress-energy tensor ensures that $R_{a b} \ell^{a} \ell^{b}=8 \pi G T_{a b} \ell^{a} \ell^{b}$ is non-negative on $\Delta$. Hence, we conclude:

$$
\sigma \bumpeq 0, \quad \text { and } \quad R_{a b} \ell^{a} \ell^{b} \bumpeq 0 .
$$

Thus, in particular, every null normal $\ell$ is free of expansion, twist and shear.

(b) Conditions on the Ricci tensor: The second equation in (III.2) implies that the vector $-R^{a}{ }_{b} \ell^{b}$ is tangential to $\Delta$. The energy condition and the field equations imply this vector must also be future causal. This means that $R_{b}^{a} \ell^{b}$ must be proportional to $\ell^{a}$ and hence, $R_{\underline{a}} \ell^{b}=0$. In the Newman-Penrose formalism this condition translates to:

$$
\Phi_{00}=\frac{1}{2} R_{a b} \ell^{a} \ell^{b} \hat{=} 0 \quad \text { and } \quad \Phi_{01}=\bar{\Phi}_{10}=\frac{1}{2} R_{a b} \ell^{a} m^{b} \hat{=} 0 .
$$

Since this statement is equivalent to $R_{a b} \ell^{b}=0$, it is gauge invariant, i.e. it does not depend upon the specific choice of null normal $\ell$ and $m$.

(c) A natural connection 1-form on $\Delta$ : Since $\ell$ is expansion, shear and twist free, there exists a one-form $\omega_{a}$ intrinsic to $\Delta$ such that

$$
\nabla_{a} \ell^{b}=\omega_{a} \ell^{b}
$$

which in turn implies

$$
\mathcal{L}_{\ell} q_{a b} \widehat{=} 2 \underset{\nabla_{a} \ell_{b}}{\widehat{=}} 0 .
$$

Thus, every null normal $\ell$ is a 'Killing field' of the degenerate metric on $\Delta$. Furthermore, we will now show that

$$
{ }^{2} \epsilon:=i m \wedge \bar{m}
$$

is also invariantly defined. Since $\mathcal{L}_{\ell} q_{a b}=0$, the space $\mathcal{S}$ of integral curves of $\ell$ is naturally equipped with a non-degenerate metric $\underline{q}_{a b}$ (so that $q_{a b}$ on $\Delta$ is the pull-back of $\underline{q}_{a b}$ ). Denote by $\underline{\epsilon}_{a b}$ the unique (up to orientation) unit alternating tensor on $\left(\mathcal{S}, \underline{q}_{a b}\right) .{ }^{2} \epsilon$ is the pull-back to $\Delta$ of $\underline{\epsilon}$. Although $\ell$ is a 'Killing field' of the intrinsic horizon geometry, the space-time metric $g_{a b}$ need not admit a Killing field in any neighborhood of $\Delta$. Robinson-Trautman metrics [15] and the Kastor-Traschen solutions [17] provide explicit examples of this type.

The 1-form $\omega$ will play an important role throughout this paper. It has an interesting geometrical interpretation. We can regard $\omega$ as a connection on the line bundle $T \Delta^{\perp}$ over $\Delta$ whose fibers are the 1-dimensional null normals to $\Delta$. Under the rescalings $\ell \mapsto \tilde{\ell}=f \ell$, of the null normal $\ell$, it transforms via:

$$
\omega_{a} \mapsto \tilde{\omega}_{a}=\omega_{a}+\nabla_{\underline{a}} \ln f .
$$


(d) Induced Connection on $\Delta$ : Each metric submanifold $M$ of $\mathcal{M}$ admits a natural connection - one which is torsion-free and compatible with the induced metric on $M$. This connection is also canonically induced by the space-time connection $\nabla$. However, since the induced metric $q_{a b}$ on $\Delta$ is degenerate, there exist infinitely many connections compatible with it. A general null sub-manifold inherits a unique (torsion-free) derivative operator $\mathcal{D}$ from $\nabla$ if and only if its null normal $\ell$ satisfies $\underline{\nabla}_{a} \ell_{b}=0$. Therefore, the conditions imposed in Definition 1 guarantee that every non-expanding horizon has a unique intrinsic derivative operator $\mathcal{D}$. The action of $\mathcal{D}$ on a vector field $X^{a}$ tangent to $\Delta$ and on a 1 -form $\eta_{a}$ intrinsic to $\Delta$ is given by

$$
\mathcal{D}_{a} X^{b} \widehat{=} \underline{\nabla}_{a} \tilde{X}^{b} \quad \text { and } \quad \mathcal{D}_{a} \eta_{b} \widehat{=} \underline{\nabla}_{a} \tilde{\eta}_{b} .
$$

where $\tilde{X}^{b}$ and $\tilde{\eta}_{b}$ are arbitrary extensions of $X^{b}$ and $\eta_{b}$ to the full space-time manifold $\mathcal{M}$. It is easy to show that $\mathcal{D}$ is independent of the extensions.

The 1 -form $\omega$ captures only part of the information in $\mathcal{D}$. The full connection $\mathcal{D}$ on $\Delta$ plays an important role in extracting physics in the strong field regime near $\Delta[18$. However, it is not essential to the discussion of isolated horizon mechanics.

(e) Conditions on the Weyl tensor: Let us begin with the definition of the Riemann tensor, $\left[\nabla_{a} \nabla_{b}-\nabla_{b} \nabla_{a}\right] X^{c}=-2 R_{a b d}^{c} X^{d}$. If we set $X^{c}=\ell^{c}$ and pull back the indices $a$ and $b$, then using (II.4), we obtain:

$$
\left[\mathcal{D}_{a} \omega_{b}-\mathcal{D}_{b} \omega_{a}\right] \ell^{c} \widehat{=}-2 R_{a b d}^{c} \ell^{d} \widehat{=}-2 C_{a b d}^{c} \ell^{d}
$$

where $C_{a b c}{ }^{d}$ is the Weyl tensor. The last equality follows from $R_{a b} \ell^{b} \widehat{=} 0$. Thus, if $v$ is any 1 -form on $\Delta$ satisfying $v \cdot \ell=0$, contracting the previous equation with $v_{c}$ we get

$$
C_{a b d}^{c} v_{c} \ell^{d} \widehat{=} 0 \text {. }
$$

Let us choose a null tetrad and set $v$ to be $m$ or $\bar{m}$. Then

$$
\Psi_{0}:=C_{a b c d} \ell^{a} m^{b} \ell^{c} m^{d} \widehat{=} 0 \quad \text { and } \quad \Psi_{1}:=C_{a b c d} \ell^{a} m^{b} \ell^{c} n^{d}=C_{a b c d} \ell^{a} m^{b} \bar{m}^{c} m^{d} \widehat{=} 0
$$

where we have used the trace-free property of the Weyl tensor in the second equation. It is also clear that equations (II.8) are independent of which null normal $\ell$, and vector fields $m$ and $\bar{m}$ we choose to construct the null tetrad; equation (II.8) is gauge invariant.

(f) Curvature of $\omega$ : Let us contract (II.7) with $n_{c}$ and use $\ell^{a} n_{a}=-1$. Then we have:

$$
2 \mathcal{D}_{[a} \omega_{b]} \widehat{=} C_{a b d} \ell^{d} n_{c} \widehat{=} C_{a b c d} \ell^{c} n^{d} .
$$

Expanding the Weyl tensor in terms of the $\Psi$ 's, one obtains

$$
\begin{aligned}
C_{a b c d} \ell^{c} n^{d} \widehat{=} & 4\left(\operatorname{Re}\left[\Psi_{2}\right]\right) n_{[a} l_{b]}+2 \Psi_{3} \ell_{[a} m_{b]}+2 \bar{\Psi}_{3} \ell_{[a} \bar{m}_{b]} \\
& -2 \bar{\Psi}_{1} n_{[a} m_{b]}-2 \Psi_{1} n_{[a} \bar{m}_{b]}+4 i\left(\operatorname{Im}\left[\Psi_{2}\right]\right) m_{[a} \bar{m}_{b]} .
\end{aligned}
$$


where

$$
\Psi_{2}: \hat{=} C_{a b c d} \ell^{a} m^{b} \bar{m}^{c} n^{d} \quad \text { and } \quad \Psi_{3}: \hat{=} C_{a b c d} \ell^{a} n^{b} \bar{m}^{c} n^{d}
$$

Substituting this expression into (II.9), pulling back on the two free indices and taking into account ([1.8) and (II.5), we obtain

$$
d \omega \widehat{=} 2\left(\operatorname{Im}\left[\Psi_{2}\right]\right)^{2} \epsilon
$$

This relation will play an important role in what follows. Note that, because $\Psi_{0}$ and $\Psi_{1}$ vanish on $\Delta, \Psi_{2}$ is gauge invariant.

Remark: It is interesting to compare the structure of $\Delta$ with that of null-infinity $\mathcal{I}$ (in the usual conformal gauge, in which the conformal factor is chosen such that the nullnormal to $\mathcal{I}$ is divergence-free). Both are null surfaces and can be regarded as 'line bundles' over a base space $\mathcal{S}$ of the integral curves of null normals. (For brevity, we will ignore a caveat concerning completeness of fibers.) The null normals are Killing fields of the intrinsic degenerate metric so that this metric is the pull-back to the 3-surface of a positive-definite metric on $\mathcal{S}$. In both cases, the space-time connection induces an intrinsic connection on the 3 -surface [19]. These connections capture physically important information. However, there are a number of differences as well. Since $\mathcal{I}$ is constructed by a conformal completion, the conformal freedom permeates all geometric structures at $\mathcal{I}$. In particular, given a physical space-time, the intrinsic metric and the derivative operator are known only up to conformal transformations. On the other hand, since $\mathcal{I}$ is at infinity, in some ways its structure is both more rigid and simpler. First, without loss of generality, we can assume that the metric on $\mathcal{S}$ is a 2 -sphere metric; the issue of distortion is physically irrelevant at $\mathcal{I}$. Second, the Weyl tensor vanishes identically at $\mathcal{I}$ and the curvature of the intrinsic connection captures non-trivial information about the next order space-time curvature. By contrast, at $\Delta$ only four components of the Weyl curvature vanish and four other components are coded in the curvature of the intrinsic connection on $\Delta$. In spite of these differences, one can carry over some techniques from null infinity to extract physical information about isolated horizons. In particular, using the analogs of techniques which have been successful at $\mathcal{I}$, one can introduce preferred cross sections of and Bondi-type expansions near $\Delta$ [18.

This concludes our analysis of the consequences of the boundary conditions defining nonexpanding horizons. Note that, even though $\ell$ is a Killing field for the intrinsic, degenerate metric $q_{a b}$ on $\Delta$, it is not an infinitesimal symmetry for other geometrical fields such as the intrinsic connection $\mathcal{D}$ or components of the curvature tensor. In the next sub-section, we will make the structure more rigid by suitably restricting the choice of $\ell$.

\section{B. Weakly Isolated horizons}

The time-independence of the intrinsic metric $q_{a b}$ captures the idea that $\Delta$ is isolated in a suitable sense. While this condition has rich consequences, the resulting structure is still 
not sufficient for physical applications. In particular, since $\ell$ can be rescaled by an arbitrary positive definite function, the acceleration $\kappa_{(\ell)}$ is not necessarily constant on $\Delta$. Therefore, we need to impose additional restrictions on the physical fields at $\Delta$ to establish the zeroth law. Since $\ell$ is already a symmetry of the intrinsic metric, it is natural to require it also be a symmetry of the 'extrinsic curvature'. However, the standard definition of the extrinsic curvature is not applicable to null surfaces. Nonetheless, given a null normal $\ell$, we can construct a tensor field $K_{a}{ }^{b}:=\mathcal{D}_{a} \ell^{b}$, defined intrinsically on $\Delta$, which can be thought of as the analogue of the extrinsic curvature. Indeed, on a metric sub-manifold, if we replace $\ell$ by the unit normal, $K_{a}{ }^{b}$ is precisely the extrinsic curvature. It is then natural to demand that, on an isolated horizon, $K_{a}{ }^{b}$ also be time-independent: $\mathcal{L}_{\ell} K_{a}{ }^{b} \widehat{=} 0$. As a consequence of (II.4), this is equivalent to imposing $\mathcal{L}_{\ell} \omega \widehat{=} 0$.

Let us examine the above condition. As we will show at the end of this section, given a non-expanding horizon we can always find a null normal $\ell^{a}$ which satisfies $\mathcal{L}_{\ell} \omega \widehat{=} 0$. The behavior of this condition under rescalings of $\ell$ is complicated by the fact that $\omega$ itself depends upon the choice of null normal (see equation (II.6)). However, under a constant rescaling $\ell \mapsto \tilde{\ell}=c \ell$, the connection 1 -form $\omega$ is unchanged. Therefore, if $\ell$ satisfies the condition $\mathcal{L}_{\ell} \omega \widehat{=} 0$, so does any $\tilde{\ell}$ related to $\ell$ by constant rescaling. This suggests we introduce an equivalence relation: Two future-directed null normals $\ell$ and $\tilde{\ell}$ belong to the same equivalence class $[\ell]$ if and only if $\tilde{\ell}=c \ell$ for some positive constant $c$.

The above considerations lead us to the following definition:

Definition 2: A weakly isolated horizon $(\Delta,[\ell])$ consists of a non-expanding horizon $\Delta$, equipped with an equivalence class $[\ell]$ of null normals to it satisfying

$$
\mathcal{L}_{\ell} \omega \widehat{=} 0 \text { for all } \ell \in[\ell] \text {. }
$$

As pointed out above, if this last equation holds for one $\ell$, it holds for all $\ell$ in $[\ell]$.

A Killing horizon (with 2-sphere cross-sections) is automatically a weakly isolated horizon, (provided the matter fields satisfy the energy condition of Definition 1). Given a nonexpanding horizon $\Delta$, one can always find an equivalence class $[\ell]$ of null-normals such that $(\Delta,[\ell])$ is a weakly isolated horizon. However, condition ([I.13) does not by itself single out the appropriate equivalence class $[\ell]$. As indicated in Section [ПD, one can further strengthen the boundary conditions and provide a specific prescription to select the equivalence class $[\ell]$ uniquely. However, for mechanics of isolated horizons, these extra steps are unnecessary.

\footnotetext{
${ }^{5}$ We are grateful to Thibault Damour for pointing out that $K_{a}{ }^{b}$ is called the Weingarten map and is analogous to extrinsic curvature. This comment suggested the above motivation for our condition on the connection 1-form $\omega$. For an alternate, and in a sense weaker, condition see the remark at the end of Section IID. From the viewpoint of intrinsic structures on $\Delta$ discussed in Section IIA, it is perhaps more natural to ask that $\ell$ be a symmetry of the full intrinsic connection $\mathcal{D}$ (see section IID and [18].) However, this stronger condition is not necessary for the laws of mechanics discussed here.
} 
In particular, our analysis will not depend on how the equivalence class $[\ell]$ is chosen. The adverb 'weakly' in Definition 2 emphasizes this point.

The condition ([1.13) has several consequences which are relevant for this paper.

(a) Surface gravity: In the case of Killing horizons $\Delta_{\mathrm{K}}$, surface gravity is defined as the acceleration of the Killing field $\xi$ normal to $\Delta_{\mathrm{K}}$. However, if $\Delta_{\mathrm{K}}$ is a Killing horizon for $\xi$, it is also a Killing horizon for $c \xi$ for any positive constant $c$. Hence, surface gravity is not an intrinsic property of $\Delta_{\mathrm{K}}$, but depends also on the choice of a specific Killing field $\xi$. (Of course the result that the surface gravity is constant on $\Delta_{K}$ is insensitive to this rescaling freedom.) In asymptotically flat space-times admitting global Killing fields, this ambiguity is generally resolved by selecting a preferred normalization in terms of the structure at infinity. For example, in the static case, one requires the Killing field $\xi$ to be unit at infinity. However, in absence of a global Killing field or asymptotic flatness, this strategy does not work and we simply have to accept the constant rescaling freedom in the definition of surface gravity. In the context of isolated horizons, then, it is natural to keep this freedom.

A weakly isolated horizon is similarly equipped with a preferred family $[\ell]$ of null normals, unique up to constant rescalings. Therefore, it is natural to interpret $\kappa_{(\ell)}$ as the surface gravity associated with $\ell$. Under the permissible rescalings $\ell \mapsto \tilde{\ell}=c \ell$, the surface gravity transforms via: $\kappa_{(\tilde{\ell})}=c \kappa_{(\ell)}$. Thus, while $\omega$ is insensitive to the rescaling freedom in $[\ell], \kappa_{(\ell)}$ captures this freedom fully. One can, if necessary, select a specific $\ell$ in $[\ell]$ by demanding that $\kappa_{(\ell)}$ be a specific function of the horizon parameters which are insensitive to this freedom, e.g., by setting $\kappa_{(\ell)}=1 / 2 R_{\Delta}$, where $R_{\Delta}$ is the horizon radius (related to the horizon area $a_{\Delta}$ via $\left.a_{\Delta}=4 \pi R_{\Delta}^{2}\right)$.

(b) Zeroth law: The boundary conditions of Definition 2 allow us to define surface gravity $\kappa_{(\ell)}$ of a weakly isolated horizon $(\Delta,[\ell])$. We will now show that the surface gravity is constant on $\Delta$. In other words, the zeroth law holds for weakly isolated horizons.

Recall from (II.12) that on a non-expanding horizon, $d \omega \hat{=} 2 \operatorname{Im}\left[\Psi_{2}\right]^{2} \epsilon$ for any choice of null normal $\ell$. Since ${ }^{2} \epsilon$ is the pull-back to $\Delta$ of the alternating tensor $\underline{\epsilon}$ on the space $\mathcal{S}$ (of orbits of $\ell$ ), clearly $\ell \cdot{ }^{2} \epsilon=0$. Therefore,

$$
\ell \cdot d \omega \widehat{=} 0
$$

for every null normal $\ell$. In particular, on a weakly isolated horizon this equation holds for any $\ell \in[\ell]$. Moreover, each of these restricted null normals also satisfies

$$
0 \widehat{=} \mathcal{L}_{\ell} \omega \widehat{=} d(\ell \cdot \omega)+\ell \cdot d \omega
$$

Hence, we conclude:

$$
d(\ell \cdot \omega) \widehat{=} d\left(\kappa_{(\ell)}\right) \widehat{=} 0,
$$

where we have used the definition ([I.1) of $\kappa_{(\ell)}$. Thus, surface gravity is constant on $\Delta$.

Although this proof of the zeroth law appears extremely simple, the argument is not as trivial as it might first appear since we have used a number of consequences of the boundary 
conditions derived in section [IA. In contrast to earlier derivations [2,10] we do not require the presence of a Killing field even in a neighborhood of $\Delta$. Therefore the proof applies also to space-times such as the Robinson-Trautman solutions [15] which do not admit a Killing field. Also, $\Delta$ need not be 'complete' — it may be of finite affine length with respect to any $\ell$ - and may not admit the analog of a 'bifurcate surface' on which the Killing field vanishes. Finally, the field equations are used rather weakly; we only need to assume that $-\left(R_{b}^{a}-\frac{1}{2} R \delta^{a}{ }_{b}\right) \ell^{b}$ is a future directed causal vector.

Surface gravity does not have a definite value on a weakly isolated horizon. The value of $\kappa_{(\ell)}$ depends upon the choice of null normal $\ell \in[\ell]$. Since all the normals $\ell$ to $\Delta$ are future directed, the rescaling constant $c$ is necessarily positive. Therefore, if the surface gravity is non-zero (respectively, zero) with respect to one $\ell$, it is non-zero (respectively, zero) with respect to any other $\ell \in[\ell]$. This rescaling freedom is the same as the one discussed above in the context of Killing horizons.

We will conclude this sub-section with three remarks.

i) Freedom in the choice of $[\ell]$ : Given a non-expanding horizon $\Delta$, it is natural to ask if one can always select an equivalence class $[\ell]$ of null normals such that $(\Delta,[\ell])$ is a weakly isolated horizon. As indicated earlier in this section, the answer is in the affirmative and, furthermore, there is a considerable freedom in the choice of $[\ell]$. Let us examine this issue in some detail.

Since $\ell \cdot d \omega \hat{=} 0$ for any null normal $\ell$ to a non-expanding horizon, it follows that a null normal $\ell$ satisfies $\mathcal{L}_{\ell} \omega \widehat{=} 0$ if and only if $d \kappa_{(\ell)} \widehat{=} 0$. Thus, to find a family [ $\left.\ell\right]$ required in the definition of weakly isolated horizons, it is necessary and sufficient to find a null normal $\ell$ for which the surface gravity is constant. On a non-expanding horizon, the surface gravity transforms as follows.

$$
\text { If } \ell \mapsto \tilde{\ell} \widehat{=} f \ell, \quad \text { then } \quad \kappa_{(\tilde{\ell})} \widehat{=} f \kappa_{(\ell)}+\mathcal{L}_{\ell} f \text {. }
$$

Hence, starting with any $\ell$, we can simply solve for $f$ by requiring that $\kappa_{(\tilde{l})}$ be constant on $\Delta$. The solution is not unique. If $\kappa_{\ell}$ is constant, given any non-zero function $g$ satisfying $\mathcal{L}_{\ell} g \widehat{=} 0$ and a constant $\tilde{\kappa}$, let us set

$$
f \widehat{=} g e^{-\kappa_{(\ell)} v}+\frac{\tilde{\kappa}}{\kappa_{(\ell)}}
$$

where $v$ satisfies $\mathcal{L}_{\ell} v \widehat{=} 1$. Then, we obtain an $\tilde{\ell} \notin[\ell]$ for which $\kappa_{(\tilde{\ell})} \widehat{=} \tilde{\kappa}$. This is the only freedom if both $\kappa_{\ell}$ and $\kappa_{(\tilde{\ell})}$ are to be constant. Thus, each non-expanding horizon gives rise to an infinite family of weakly isolated horizons. Put differently, although one can easily obtain weakly isolated horizons from non-expanding ones by choosing appropriate null normals $[\ell]$, a specific weakly isolated horizon carries much more information than the non-expanding horizon it comes from. At the end of Section IID, we will indicate how one can further strengthen the boundary conditions to give a prescription for selecting a specific $[\ell]$. However, the analysis of this paper does not depend on how this selection is made.

ii) How does Definition 2 compare with that used in the undistorted, non-rotating case? As one would expect, the definition given in [6 8] is significantly stronger. Furthermore, it 
was tied to a foliation from the beginning. More precisely, it assumed that there exists a foliation to which $\omega$ is normal, with $\omega=-\kappa n$, and it required that the expansion $\operatorname{Re}[\mu]$ of the null normal $n$ to the leaves of the foliation be constant on $\Delta$ (see appendix $\mathbb{B}$ for definitions of the NP spin coefficients). Although it was shown that the foliation is unique if it exists, the heavy reliance on the foliation right from the beginning made that definition less elegant and its invariant content less transparent. Also, since we now allow $d \omega$ to be non-zero and impose no conditions on $\operatorname{Re}[\mu]$, we can now incorporate rotation and distortion.

iii) Alternate boundary conditions: In the definition of isolated horizons, we required $\mathcal{L}_{\ell} \omega \widehat{=}$, which in particular implies $\mathcal{L}_{\ell} \kappa_{(\ell)} \equiv \mathcal{L}_{\ell}(\ell \cdot \omega) \widehat{=} 0$. Thus, the definition itself assured us that $\kappa_{(\ell)}$ is 'time independent' and to prove the zeroth law we had to show that it is also independent of 'angles'. Could we have used another definition in which the 'time dependence' of $\kappa_{(\ell)}$ was not explicitly required but followed from other conditions? The answer is in the affirmative: In place of $\mathcal{L}_{\ell} \omega \widehat{=} 0$, we could have required that $\Delta$ admit a foliation on which the expansion $\operatorname{Re}[\mu]$ of $n$ and the Newman-Penrose spin coefficient $\pi$ which carries the angular momentum information are 'time independent' [13]. Then the field equations would have implied that $\kappa_{(\ell)}$ is time-independent. Furthermore, all results of this paper go through (and were in fact first obtained) with these modified boundary conditions. Note however that the new condition is neither weaker nor stronger than the one we used. Both require that $\pi$ be time independent. In addition, the present definition of isolated horizons requires that $\kappa_{(\ell)}$ be time independent while the alternative definition would have required, instead, that $\operatorname{Re}[\mu]$ be time independent. In this paper, we chose the present definition because it can be stated without reference to a foliation.

\section{Electromagnetic Field}

We shall now describe the form of the electromagnetic field at an isolated horizon and introduce a partial gauge fixing at the horizon which will allow us to introduce the notion of an electric potential. In the next three sections - where we discuss the action, phase space and first law — we will assume that the only matter field present at the isolated horizon are Maxwell fields. However, as our discussion will make it clear, this restriction is made primarily for simplicity. The overall framework is rather general and can accommodate matter for which there exists a well-defined action principle and a (covariant) Hamiltonian framework. In particular, in Section VI, we describe how to extend the formalism to include Yang Mills Fields.

The isolated horizon boundary conditions restrict matter primarily through conditions on the stress-energy tensor $T_{a b}$. Let us begin with $T_{a b} \ell^{a} \ell^{b} \hat{=} 0$, a direct consequence of the boundary conditions and Raychaudhuri equation. (This restriction arises due to the fact that $\Delta$ is a non-expanding horizon; the subsequent stronger boundary conditions do not further constrain F). Although this condition is weak, it turns out to have interesting consequences on the form of the electromagnetic field, $\mathbf{F}$, at $\Delta$. The stress-energy tensor for electromagnetism is given in terms of the field strength $\mathbf{F}$ as 


$$
\mathbf{T}_{a b}=\frac{1}{4 \pi}\left[\mathbf{F}_{a c} \mathbf{F}_{b}^{c}-\frac{1}{4} g_{a b} \mathbf{F}_{c d} \mathbf{F}^{c d}\right]
$$

Let us contract this expression with $\ell^{a} \ell^{b}$ and examine the consequences for $\mathbf{F}$. This gives

$$
0 \widehat{=} \mathbf{T}_{a b} \ell^{a} \ell^{b} \widehat{=}\left|\ell^{a} m^{b} \mathbf{F}_{a b}\right|^{2}
$$

where, to obtain the last expression, we have used the anti-symmetry of $\mathbf{F}$ and the fact that the metric at the horizon can be expressed in terms of a null-tetrad as $g_{a b}=-2 \ell_{(a} n_{b)}+$ $2 m_{(a} \bar{m}_{b)}$. An immediate consequence of (II.15) is that

$$
\ell^{a} \mathbf{F}_{a b} \widehat{=} 0 \text {. }
$$

In order to obtain a similar expression for ${ }^{\star} \mathbf{F}$ recall that the stress energy tensor can be rewritten as $\mathbf{T}_{a b}=-\frac{1}{4 \pi}\left[{ }^{\star} \mathbf{F}_{a c}{ }^{\star} \mathbf{F}_{b}{ }^{c}-\frac{1}{4} g_{a b}{ }^{\star} \mathbf{F}_{c d}{ }^{\star} \mathbf{F}^{c d}\right]$. Applying the same argument which led to (II.16), we obtain a similar restriction on ${ }^{\star} \mathbf{F}$, namely

$$
\ell^{a \star} \mathbf{F}_{a b} \widehat{=} 0 \text {. }
$$

These two restrictions tell us there is no flux of electromagnetic radiation across the horizon.

It is straightforward to show that ([1.16), (II.17) and the form of the metric at $\Delta$ place further restrictions on $\mathbf{T}_{a b}$ :

$$
\begin{aligned}
& \mathbf{T}_{a b} \ell^{a} m^{b} \hat{=} 0 \quad \mathbf{T}_{a b} \ell^{a} \bar{m}^{b} \hat{=} 0 \\
& \mathbf{T}_{a b} m^{a} m^{b} \hat{=} 0 \quad \mathbf{T}_{a b} \bar{m}^{a} \bar{m}^{b} \hat{=} 0 .
\end{aligned}
$$

The first two equations contain no new information since we already knew from general arguments (see equation ([1.3)), that $\Phi_{10} \widehat{=} 0$ and $\Phi_{01} \widehat{=} 0$. However, the last two equations do place further restrictions on the stress energy tensor. Since the equations of motion are enforced at the boundary, we see immediately that they are equivalent to further restricting the Ricci tensor at the horizon by requiring:

$$
\Phi_{02}:=\frac{1}{2} R_{a b} m^{a} m^{b} \hat{=} 0 \quad \text { and } \quad \Phi_{20}:=\frac{1}{2} R_{a b} \bar{m}^{a} \bar{m}^{b} \widehat{=} 0 .
$$

This result need not hold for general matter fields; it relies on the properties of the electromagnetic stress-energy tensor (II.14).

The next task is to define the electric and magnetic charges of the horizon. Since the horizon is an inner boundary of spacetime, the normal to a 2-sphere cross section of the horizon will naturally be inward pointing. Bearing this in mind, we define the electric and magnetic charges of the horizon as

$$
Q_{\Delta}:=-\frac{1}{4 \pi} \oint_{S_{\Delta}}{ }^{\star} \mathbf{F} \quad \text { and } \quad P_{\Delta}:=\frac{1}{4 \pi} \oint_{S_{\Delta}} \mathbf{F} \text {. }
$$

For these definitions to be meaningful, the values of $Q_{\Delta}$ and $P_{\Delta}$ should be independent of the cross section of the horizon $S_{\Delta}$. We will now show that $\Delta$ being a non-expanding horizon guarantees this is the case. Let us first evaluate 


$$
\mathcal{L} \ell_{\leftarrow} \mathbf{F} \widehat{=} \ell \cdot d \mathbf{F}+\underset{\longleftarrow}{d(\ell \cdot \mathbf{F})} .
$$

The first term on the right hand side vanishes due to Maxwell's equations on $\Delta$, while the second term is zero due to the previous restriction on $\mathbf{F}$, (II.16). Therefore we conclude that $\mathbf{F}$ is Lie dragged by $\ell$. An identical argument for ${ }^{\star} \mathbf{F}$ leads to the analogous conclusion. Therefore we obtain

$$
\mathcal{L}_{\ell} \mathbf{F}=0 \quad \text { and } \quad \mathcal{L}_{\ell_{\leftarrow}^{\star}} \mathbf{F}=0 \text {. }
$$

This result, along with ([I.16) and (ㅍ.17), guarantees that $Q_{\Delta}$ and $P_{\Delta}$ are independent of the choice of cross section $S_{\Delta}$ of the horizon. Note that this result was obtained using only the boundary conditions; equations of motion in the bulk are not needed.

Finally, let us examine the remaining freedom in the electromagnetic field. The boundary conditions do not restrict $\mathbf{F}_{a b} n^{a} m^{b}$ and ${ }^{\star} \mathbf{F}_{a b} n^{a} m^{b}$ at all. These components describe the electromagnetic radiation flowing along the horizon. Therefore, isolated horizon boundary conditions allow electromagnetic radiation arbitrarily close to - and even at - the horizon, provided none crosses it.

So far we have confined ourselves to the field strengths $\mathbf{F}$ and ${ }^{\star} \mathbf{F}$. However, in the action principle and the Hamiltonian framework we have to consider also the Maxwell potential $\mathbf{A}$. Now, if the magnetic charge is non-zero, either one has to allow 'wire singularities' in the vector potentials or regard $\mathbf{A}$ as a connection on a non-trivial $U(1)$-bundle. (If we regard it as a connection on a $\mathbb{R}^{+}$-bundle, the magnetic charge is necessarily zero.) Since we wish to deal only with smooth fields, we will not allow 'wire singularities' in the potentials. If we work with bundles, the magnetic charge is quantized whence the space of histories has several disconnected components. Thus, in the first law, we will not be able to consider variations $\delta$ of fields with $\delta P \neq 0$. As far as mechanics of isolated horizons is concerned, there is essentially no loss of generality if we restrict ourselves to the case $P_{\Delta}=0$. Therefore, in the next three sections, while working with Maxwell fields, we will do so. As usual, our final results can be formally extended to the case of non-vanishing magnetic charge by performing a duality rotation on F. (As discussed in Section V1, the situation is rather different in the case of Yang-Mills fields.)

Recall that the first law in the Einstein-Maxwell case involves the electro-static potential $\Phi$. In static space-times, one typically sets $\Phi=-\xi^{a} \mathbf{A}_{a}$ where $\xi$ is the static Killing field and the gauge is chosen such that the vector potential $\mathbf{A}$ tends to zero at infinity and satisfies $\mathcal{L}_{\xi} \mathbf{A}=0$ everywhere in space-time. We now need a strategy to define the electric potential $\Phi$ without reference to a Killing field or infinity. To this end, we introduce the following definition:

Definition 3: The electromagnetic potential $\mathbf{A}$ will be said to be in a gauge adapted to the weakly isolated horizon $(\Delta,[\ell])$ if it satisfies

$$
\mathcal{L}_{\ell} \stackrel{\mathbf{A}}{=} 0 \text {. }
$$

Mathematically, this restriction is analogous to this condition $\mathcal{L}_{\ell} \omega \widehat{=} 0$ imposed on the gravitational field in Definition 2. However, while the condition on $\omega$ is a physical restriction 
on the form of the gravitational field at $\Delta$, the condition on $\mathbf{A}$ is a gauge choice; it can always be imposed without physically constraining the electromagnetic field strength.

Given an electromagnetic potential $\mathbf{A}$ in a gauge adapted to $(\Delta,[\ell])$, we can now define the scalar potential $\Phi_{(\ell)}$ at the horizon in an obvious fashion:

$$
\Phi_{(\ell)}: \widehat{=}-\ell \cdot \mathbf{A} \text {. }
$$

The key question now is whether our boundary conditions are strong enough to ensure that $\Phi_{(\ell)}$ is constant on $\Delta$. Only then can we hope to use this notion of the scalar potential in the first law. Note that this question is rather similar to the one we asked of surface gravity $\kappa_{(\ell)}$ in Section IIB. By using arguments completely analogous to those that led us to the zeroth law, we will now show that the answer to the present question is also in the affirmative. In a gauge adapted to the horizon,

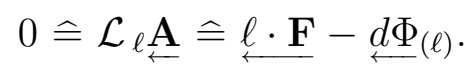

As we saw above, the boundary conditions imply $\ell \cdot \mathbf{F} \widehat{=} 0$ (equation (II.16)). Hence, it follows immediately that $\Phi_{(\ell)}$ is constant on the horizon. We can regard this result as the 'electromagnetic part' of the zeroth law of isolated horizon mechanics.

We will see in the next section that these zeroth laws play a key role in making the gravitational and the electromagnetic action principles well-defined in presence of isolated horizons. As with surface gravity $\kappa_{(\ell)}$, the functional dependence of $\Phi_{(\ell)}$ on the horizon parameters varies with the choice of $\ell \in[\ell]$. We will see in Section $\nabla$ that the Hamiltonian framework constrains these dependencies in an interesting fashion.

\section{Other definitions and remarks}

In this sub-section, we introduce two new definitions which are important to the general framework of isolated horizons.

The first is concerned with rotation. From one's experience with the Newman-Penrose framework, one expects the gravitational contribution to angular momentum to be coded in the imaginary part of $\Psi_{2}$. This expectation will be shown to be correct in [13]. Therefore, in the Einstein-Maxwell theory, we introduce the following definition:

Definition 4: A weakly isolated horizon $(\Delta,[\ell])$ will be said to be non-rotating if $\operatorname{Im}\left[\Psi_{2}\right]$ vanishes on $\Delta$.

If $\left(\Delta_{K},[\xi]\right)$ is a Killing horizon and $\xi$ is a hypersurface orthogonal, time-like vector field near $\Delta_{\mathrm{K}}$, on physical grounds one would expect the horizon to be non-rotating. Is this expectation compatible with our definition? The answer is in the affirmative. For, in this case, one can show that $B_{a b}:=C_{a c b d} \xi^{c} \xi^{d}$ vanishes in the region where $\xi^{a}$ is time-like. Hence, by continuity, it also vanishes on $\Delta$ forcing $\operatorname{Im}\left[\Psi_{2}\right]$ to vanish there. Similarly, if the space-time admits a hypersurface orthogonal, rotational Killing field $\varphi^{a}$ in a neighborhood of $\Delta \operatorname{Im}\left[\Psi_{2}\right]$ again 
vanishes on $\Delta$. The definition is again compatible with one's intuition that the horizon should be non-rotating in this case. In this paper, while we allow for presence of rotation in the first four sections, we will restrict ourselves to non-rotating horizons in the proof of the first law in Section V.

Finally, for completeness, let us introduce a stronger notion of 'isolation' by strengthening the boundary conditions of Definition 2.

Definition 5: A weakly isolated horizon $(\Delta,[\ell])$ is said to be isolated if

$$
\left[\mathcal{L}_{\ell}, \mathcal{D}\right] V \widehat{=} 0
$$

for all vector fields $V$ tangential to $\Delta$ and all $\ell \in[\ell]$

As before, if any one $\ell$ satisfies this condition, so do all $\ell \in[\ell]$. However, unlike (II.13), condition ([I.22) is a genuine restriction in the sense that it can not always be met by a judicious choice of null normals. Generically it does suffice to single out the equivalence class

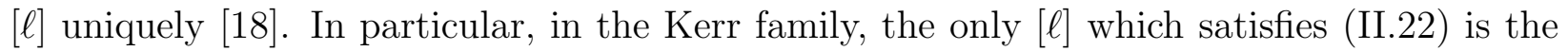
one containing constant multiples of the globally defined Killing field which is orthogonal to the horizon. Every Killing horizon is of course an isolated horizon. Thus, even though (I1.22) is a stronger condition than (II.13), it is still very weak compared to conditions normally imposed. For most physical applications, e.g., to numerical relativity, it is appropriate to work with isolated horizons. For mechanics of isolated horizons, however, we can - and will — work with the larger class of weakly isolated horizons.

The following consequences of the boundary conditions defining weakly isolated horizons, derived in this section, will play an important role in the subsequent discussion:

a) $\nabla_{a} \ell_{b} \widehat{=} \omega_{a} \ell^{b}$

b) $\kappa_{(\ell)} \widehat{=} \ell^{a} \omega_{a}$, the surface gravity defined by the null normal $\ell^{a}$, is constant on $\Delta$;

c) There is a natural (area) 2-form ${ }^{2} \epsilon$ on $\Delta$ satisfying $\mathcal{L}_{\ell}{ }^{2} \epsilon \widehat{=} 0$ and ${ }^{2} \epsilon_{a b} \ell^{b} \widehat{=} 0$.

d) The electromagnetic potential $\mathbf{A}$ is chosen to satisfy $\mathcal{L}_{\ell} \mathbf{A} \widehat{=} 0$ and in this gauge the scalar potential $\Phi_{(\ell)}:=-\ell^{a} \mathbf{A}_{a}$ is constant on $\Delta$; and

e) The electromagnetic field satisfies $\ell^{\ell^{a} \mathbf{F}_{a b}} \widehat{=} 0$; and $\ell^{\ell^{a \star}} \mathbf{F}_{a b} \widehat{=} 0$.

\section{ACTION}

In this paper we use the first order formulation of general relativity in terms of tetrads and connections. Since tetrads are essential to incorporate spinorial matter, it is natural to base the framework on tetrads from the beginning. The use of a first order formalism, on the other hand, is motivated primarily by mathematical simplicity. In the first order framework, the action and the Hamiltonians can be expressed entirely in terms of differential forms which significantly simplify the variational calculations. The previous paper [8] which dealt 
with undistorted horizons used spinors and self-dual connections, while here we choose to use orthonormal tetrads and real, Lorentz connections. For analyzing mechanics of isolated horizons, there are two advantages to this. First, the Hamiltonian and symplectic structure are now manifestly real which simplifies evaluation of the boundary terms at the horizon. Second, the analysis can now be extended to other space-time dimensions in a straightforward manner. However, these simplifications, come with a price. Since, at present, the self dual variables appear to be indispensable for non-perturbative quantization, the results obtained here will have to be re-expressed in terms of self-dual variables in order to extend the analysis [20] of the quantum horizon geometry and black hole entropy to include rotation.

\section{A. Preliminaries}

Let us begin with the first order action for Einstein-Maxwell theory on a 4-dimensional manifold $\mathcal{M}$ which is topologically $M \times \mathbb{R}$, where $M$ is an oriented Riemannian 3-manifold without boundary (the complement of a compact set of) which is diffeomorphic to (the complement of a compact set of) $\mathbb{R}^{3}$. Thus, topological complications of $M$, if any, are confined to a compact set. In this subsection we shall only give the relevant formulae. For details, see e.g. [21]. Our basic fields will consist of a triplet $\left(e_{a}^{I}, A_{a I}^{J}, \mathbf{A}_{a}\right)$ defined on $\mathcal{M}$ where $e_{a}^{I}$ denotes a co-tetrad, $A_{a I}{ }^{J}$ the gravitational (Lorentz) connection and $\mathbf{A}_{a}$ the electromagnetic connection. Here, lower case latin letters refer to the tangent space of $\mathcal{M}$ while the upper case letters $I, J$ etc. refer to an internal four dimensional vector space $V$ with a fixed metric $\eta_{I J}$ of signature $(-+++)$. The co-tetrad $e_{a}^{I}$ is an isomorphism between the tangent space $T_{p}(\mathcal{M})$ at any point $p$ and the internal space $V$. Using it, we define a metric on $\mathcal{M}$ by $g_{a b}:=e_{a}^{I} e_{b}^{J} \eta_{I J}$ which also has signature $(-+++)$. The Lorentz connection $A_{a I}{ }^{J}$ acts only on internal indices and defines a derivative operator

$$
D_{a} k_{I}:=\partial_{a} k_{I}+A_{a I}^{J} k_{J}
$$

where $\partial$ is a fiducial derivative operator which, as usual, will be chosen to be flat and torsion free. Finally, $\mathbf{A}_{a}$ is the $U(1)$ electromagnetic connection 1-form on $\mathcal{M}$. (As noted in Section IIQ, we will assume that the magnetic charge is zero.) All fields will be assumed to be smooth and satisfy the standard asymptotic conditions at infinity.

The 2-forms $\Sigma^{I J}$

$$
\Sigma_{I J}:=\frac{1}{2} \epsilon_{I J K L} e^{K} \wedge e^{L}
$$

constructed from the co-tetrads will play an important role throughout our calculations. In particular, the action for an asymptotically flat space-time (with no internal boundary) is given by (see e.g. 21])

$$
S(e, A, \mathbf{A})=\frac{-1}{16 \pi G} \int_{\mathcal{M}} \Sigma^{I J} \wedge F_{I J}+\frac{1}{16 \pi G} \int_{\tau_{\infty}} \Sigma^{I J} \wedge A_{I J}-\frac{1}{8 \pi} \int_{\mathcal{M}} \mathbf{F} \wedge{ }^{\star} \mathbf{F} .
$$


Here $F$ and $\mathbf{F}$ are the curvatures of the gravitational and electromagnetic connections $A$ and A respectively:

$$
F_{I}^{J}=d A_{I}^{J}+A_{I}^{K} \wedge A_{K}^{J} \quad \text { and } \quad \mathbf{F}=d \mathbf{A},
$$

${ }^{\star} \mathbf{F}_{a b}=\frac{1}{2} \epsilon_{a b}{ }^{c d} \mathbf{F}_{c d}$ is the dual of $\mathbf{F}$ defined using $e_{a}{ }^{I}$, and $\tau_{\infty}$ is the time-like cylinder at infinity. The boundary term at $\tau_{\infty}$ ensures the differentiability of the action.

Let us briefly examine the equations of motion arising from the action. Varying the action with respect to the connection, one obtains

$$
D \Sigma=0 .
$$

This condition implies that the connection $D$ defined by $A$ has the same action on internal indices as the unique connection $\nabla$ compatible with the co-tetrad, i.e., satisfying $\nabla_{a} e_{b}^{I}=0$. When this equation of motion is satisfied, the curvature $F$ is related to the Riemann curvature $R$ of $\nabla$ by

$$
F_{a b}^{I J}=R_{a b}^{c d} e_{c}^{I} e_{d}^{J}
$$

Varying the action with respect to $e_{a}^{I}$ and taking into account the above relation between curvatures, one obtains Einstein's equations

$$
G_{a b}=8 \pi G T_{a b} \equiv 2 G\left(\mathbf{F}_{a c} \mathbf{F}_{b d} g^{c d}-\frac{1}{4} g_{a b} \mathbf{F}_{c d} \mathbf{F}^{c d}\right)
$$

where $G_{a b}$ is the Einstein tensor and $T_{a b}$ the electromagnetic stress energy tensor. Finally, variation with respect to the electromagnetic connection, A, yields Maxwell's equation

$$
d^{\star} \mathbf{F}=0 .
$$

\section{B. Internal boundary $\Delta$}

Let us now consider the variational principle for asymptotically flat histories which admit a weakly isolated horizon $\Delta$ as their internal boundary. The manifold $\mathcal{M}$ under consideration has an internal boundary $\Delta$, topologically $S^{2} \times \mathbb{R}$. As before, $\mathcal{M}$ is topologically $M \times \mathbb{R}$, where $M$ is now an oriented manifold with an internal, 2-sphere boundary, whose topological complications are again confined to a compact region. Space-time is bounded to the future and past by two (partial Cauchy) surfaces $M^{ \pm}$, extending to spatial infinity (see Figure 1).

Following Definition 2 of weakly isolated horizons, we will equip $\Delta$ with a fixed equivalence class of vector fields $[\ell]$ which are transversal to its 2 -sphere cross-sections (where, as before, $\ell \sim \ell^{\prime}$ if and only if $\ell \widehat{=} c \ell^{\prime}$ for a constant $c$ ). It is also convenient to fix an internal null tetrad $\left(\ell^{I}, n^{I}, m^{I}, \bar{m}^{I}\right)$ on $\Delta$, each element of which is annihilated by the fiducial, flat internal connection $\partial$. 


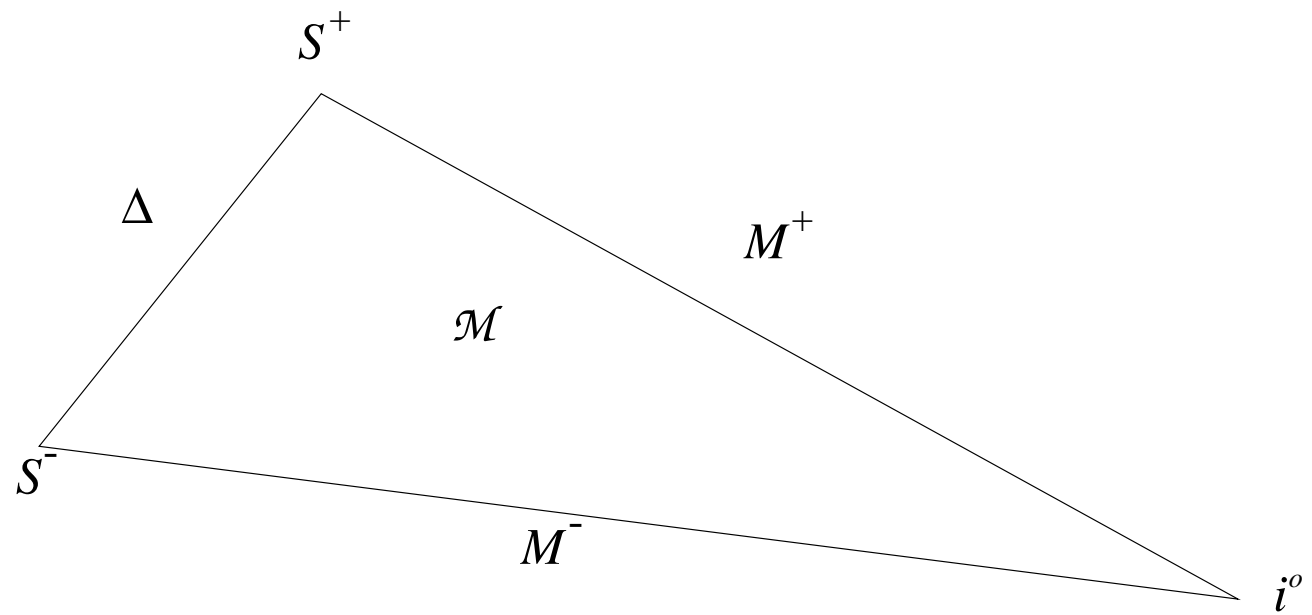

FIG. 1. The region of space-time $\mathcal{M}$ under consideration has an internal boundary $\Delta$ and is bounded by two partial Cauchy surfaces $M^{ \pm}$which intersect $\Delta$ in the 2-spheres $S^{ \pm}$and extend to spatial infinity $i^{\circ}$.

The permissible histories consist of smooth triplets $(e, A, \mathbf{A})$ on $\mathcal{M}$ satisfying boundary conditions at infinity and on $\Delta$. The boundary conditions at infinity are, as before, the standard ones which ensure asymptotic flatness. Since the asymptotic behavior and boundary integrals at infinity play only a secondary role in our analysis, we shall not spell out the precise fall-off requirements. At $\Delta$, the histories are subject to three conditions: i) the tetrads $e$ should be such that the vector field $\ell^{a}:=\ell^{I} e_{I}^{a}$ defined by each history belongs to the equivalence class $[\ell]$ fixed on $\Delta$; ii) the tetrad $e$ and the gravitational connection $A$ should be such that $(\Delta,[\ell])$ is a weakly isolated horizon for the history; and, iii) the electromagnetic potential $\mathbf{A}$ is in a gauge adapted to the horizon, i.e., $\mathcal{L}_{\ell} \mathbf{A} \widehat{=} 0$.

Remark: In space-time, we have the freedom to perform a local, internal Lorentz rotation on the tetrad $e_{I}^{a}$ (and the gravitational connection $A_{a I}{ }^{J}$ ). All these tetrads define the same Lorentzian metric $g_{a b}$. Since $\ell^{a}$ is required to be a null normal to $\Delta$, the permissible gauge rotations are reduced on $\Delta$ to the sub-group $\left(\mathbb{R}^{+} \times E^{2}\right)_{\text {loc }}$ of local null rotations preserving the null direction field $\ell$. (Here $\mathbb{R}^{+}$is the group of rescalings of $\ell^{a}, n^{a}$ which leaves $m^{a}$ fixed and $E^{2}$ is the 3 -dimensional Euclidean group consisting of rotations in the $\ell-m, \ell-\bar{m}$ and $m-\bar{m}$ planes.) Condition i) above - dictated by the existence of a preferred equivalence class $[\ell]$ in Definition 2 - further reduces the internal gauge freedom to $\mathbb{R}^{+} \times\left(E_{\mathrm{loc}}^{2}\right)$, i.e., reduces the group $\mathbb{R}_{\text {loc }}^{+}$of local $\ell-n$ rescalings to the group $\mathbb{R}^{+}$of global rescalings. Thus, while any one space-time $\left(\mathcal{M}, g_{a b}\right)$, still defines infinitely many histories due to the freedom of tetrad-rotations, this freedom is somewhat reduced at $\Delta$ because of the structure fixed by the boundary conditions.

\footnotetext{
${ }^{6}$ Nonetheless, from a space-time perspective, the multiplicity of histories can still be rather sur-
} 
Given any tetrad $e_{I}^{a}$, the internal null vectors $\left(\ell^{I}, n^{I}, m^{I}, \bar{m}^{I}\right)$ fixed on $\Delta$ trivially provide a null tetrad $\left(\ell^{a}, n^{a}, m^{a}, \bar{m}^{a}\right)$. In terms of these vectors, we can express $\Sigma_{\leftarrow}^{I J}$ as:

$$
\sum_{\leftarrow}^{I J} \widehat{=} 2 \ell^{[I} n^{J] 2} \epsilon+2 n \wedge\left(i m \ell^{[I} \bar{m}^{J]}-i \bar{m} \ell^{[I} m^{J]}\right),
$$

where, as before, ${ }^{2} \epsilon=i m \wedge \bar{m}$ is the pull-back to $\Delta$ of the natural alternating tensor on the 2 -sphere $\mathcal{S}$ of integral curves of $\ell^{a}$ associated with the given history. The weak isolation of $(\Delta,[\ell])$ restricts the form of the connection $A$ at $\Delta$. To see this, recall that one of the equations of motion requires the connection $D$ defined by $A$ to have the same action on internal indices as $\nabla$. Hence, $\nabla_{a} \ell_{I} \widehat{=} \partial_{a} \ell_{I}+A_{a}{ }^{J} \ell_{J} \widehat{=} A_{a I}{ }^{J} \ell_{J}$ where, in the second step we have used the fact that the flat derivative operator, $\partial$, has been chosen to annihilate the internal tetrad on $\Delta$. Since $\nabla_{a} e_{b}^{I}=0$ by definition of $\nabla$, and $\nabla_{a} \ell^{b} \hat{=} \omega_{a} \ell^{b}$ (see [1.4) it follows that $\underset{\leftarrow}{A} \ell_{J} \ell^{\widehat{=}} \omega \ell_{I}$. Hence, on $\Delta, \underset{\leftarrow}{A}$ has the form:

$$
\stackrel{A_{I J}}{=}-2 l_{[I} n_{J]} \omega+C_{I J},
$$

where the 1-form $C_{I J}$ satisfies $C_{I J} \ell^{J} \widehat{=} 0$.

With this background material at hand, we are now ready to consider variations of the action ([II.1) in the presence of an inner boundary representing a weakly isolated horizon. A key question is whether a new surface term at the horizon is necessary to make the variational principle well-defined. We will show that, thanks to the zeroth law, such a term is not needed.

A simple calculation yields:

$$
\delta S(e, A, \mathbf{A})=\int_{\mathcal{M}} \text { Equations of Motion } \cdot \delta \phi-\frac{1}{16 \pi G} \int_{\Delta} \Sigma^{I J} \wedge \delta A_{I J}-\frac{1}{4 \pi} \int_{\Delta} \delta \mathbf{A} \wedge{ }^{\star} \mathbf{F}
$$

where, in the first term, $\phi$ stands for the basic fields $(e, A, \mathbf{A})$ in the action. Note that, as in the case without an internal boundary, the variation of the boundary term at infinity precisely cancels the contribution arising from the variation of the bulk terms.

In order to show that the action principle is viable, it is necessary to show that the terms at the horizon vanish due to the boundary conditions imposed there. Let us begin with the gravitational term. Using ([1.2) and ([I..3) it can be re-expressed as

$$
-\frac{1}{8 \pi G} \int_{\Delta} \delta \omega \wedge^{2} \epsilon
$$

Since ${ }^{2} \epsilon$ is the pull-back to $\Delta$ of the alternating tensor on the 2-sphere $\mathcal{S}$ of integral curves of $\ell$, it follows that $\mathcal{L}_{\ell}^{2} \epsilon=0$. Furthermore, the weak isolation of the horizon ensures $\mathcal{L}_{\ell} \omega \widehat{=} 0$

prising. For example, if $g_{a b}$ is the Schwarzschild metric with mass $M>0$, there is a history in which the surface gravity $\kappa_{(\ell)}$ is positive and another in which it is zero. This redundancy can be eliminated by working with isolated, rather than weakly isolated horizons. 
and, since the null normal $\ell^{a}$ defined by any tetrad belongs to the fixed equivalence class $[\ell]$ at the horizon, we have $\delta \ell=c_{\delta} \ell$ for some constant $c_{\delta}$. These two facts imply $\mathcal{L}_{\ell} \delta \omega \hat{=} 0$. Thus the entire integrand is Lie dragged by $\ell$. In the variational principle, however, all fields are fixed on the initial and final hypersurfaces, say $M^{ \pm}$. In particular, $\delta \omega$ necessarily vanishes on the initial and final cross sections of the horizon. Therefore, the integrand in (III.5) vanishes on the initial and final cross sections and is Lie dragged by $\ell$. This immediately implies (III.5) is zero.

Let us now consider the electromagnetic term. Since every $\mathbf{A}$ is in a gauge adapted to the isolated horizon, $\mathcal{L}_{\ell} \mathbf{A} \bumpeq 0$. Furthermore, $\delta \ell^{a} \bumpeq c_{\delta} \ell^{a}$, so we conclude $\mathcal{L}_{\ell} \delta \mathbf{A} \cong 0$. Next, (【I.20) ensures $\mathcal{L}_{\ell}{ }^{\star} \mathbf{F}=0$. Thus, the integrand of the electromagnetic surface term is Lie dragged by $\ell^{a}$. An identical argument to the one presented above implies that the electromagnetic surface term in (III.4) also vanishes. Therefore, the variation of the action (IIII.1) continues to yield Einstein-Maxwell equations in spite of the presence of an inner boundary representing a weakly isolated horizon.

It is instructive to re-examine the key step in the above argument. Suppose we only had a non-expanding horizon. Then, the gravitational surface term could still be reduced to (III.5), and ${ }^{2} \epsilon$ and ${ }^{\star} \mathbf{F}$ would still be Lie-dragged by $\ell$. However, in this case, we could not argue that $\omega$ and $\mathbf{A}$ are also Lie-dragged. As we saw in Sections $\Pi \mathrm{IB}$ and $\mathbb{\mathrm { II }}$, these conditions are equivalent, respectively, to the constancy of the surface gravity $\kappa_{(\ell)}$ and the electromagnetic potential $\Phi_{(\ell)}$ on $\Delta$. In this sense, given a non-expanding horizon as the inner boundary, the gravitational and electromagnetic zeroth laws are the necessary and sufficient conditions one must impose for the viability of the standard, first order, tetrad action principle.

Remark: Note that (IIII.1) is not the unique viable action for the problem: as usual, there is freedom to add suitable boundary terms without affecting the viability. Specifically, we are free to add any horizon boundary term which is composed entirely of fields which are Lie dragged by $\ell$, for example the intrinsic horizon metric $q_{a b}$ and fields $\omega,{ }^{2} \epsilon$, and $\underset{\leftarrow}{\mathbf{A}}$. Then, due to the argument given above, the new action would also be viable. However, as usual, this freedom will not affect the definition of the symplectic structure which underlies the Hamiltonian treatment of the next section.

\section{COVARIANT PHASE SPACE}

Let us now construct the phase space of space-times containing weakly isolated horizons. In the next section, we will use this framework to construct Hamiltonians generating suitable time translations and define the energy of an isolated horizon. In [8], the phase space was constructed by performing a Legendre transform of the action. This procedure leads to a 'canonical' framework in which the phase space consists of configuration and momentum variables defined on a spatial hypersurface. With the self-dual connections used in [8], the gravitational configuration variable turns out to be a connection and its conjugate momentum, a 2-form so that the Hamiltonian description can again be given in terms of forms. With the full Lorentz connections now under consideration, the situation turns out 
to be more complicated. Specifically, one encounters certain second class constraints and, when these are solved, one ends up with the same canonical phase space that one would have obtained through a second order formalism. In the Hamiltonian framework, then, the simplicity we encountered in Section III is lost. More specifically, constraint functions and Hamiltonians now contain terms involving second derivatives of the basic canonical variables which make variations rather complicated. (For details, see chapters 3 and 4 in 21 .) Therefore, in this section we will not use a Legendre transform. Instead, we will construct the 'covariant phase space' from the space of solutions to field equations (see, e.g., [22]). As in Section III, all expressions will now involve only the basic form-fields and their exterior derivatives and variational calculations will continue to be simple.

To specify the phase space, let us begin as in Section III by fixing a manifold $\mathcal{M}$ with an internal boundary $\Delta$ (see figure 1). As before, we will equip $\Delta$ with an equivalence class $[\ell]$ of vector fields transverse to its 2 -sphere cross-sections. To evaluate the symplectic structure and Hamiltonians, we will often use a partial Cauchy surface $M$ in the interior of $\mathcal{M}$ which intersects $\Delta$ in a 2 -sphere $S$. Points of the covariant phase space $\Gamma$ will consist of histories considered in Section $\mathbb{I I}$ which satisfy field equations. More explicitly, $\Gamma$ consists of asymptotically flat solutions $(e, A, \mathbf{A})$ to the field equations on $\mathcal{M}$ such that i) the vector field $\ell^{a}:=\ell^{I} e_{I}^{a}$ belongs to the equivalence class $[\ell]$ fixed on $\Delta$, ii) in each solution, $(\Delta,[\ell])$ is a weakly isolated horizon; and, iii) the electromagnetic potential $\mathbf{A}$ is in a gauge adapted to the horizon, i.e., $\mathcal{L}_{\ell} \mathbf{A}=0$.

Our next task is to use the action ([II.1) to define the symplectic structure $\Omega$ on $\Gamma$. It is convenient to make a brief detour and first introduce two new fields which can be regarded as 'potentials' for the surface gravity $\kappa_{(\ell)}$ and the electric potential $\Phi_{(\ell)}$. Given any point $(e, A, \mathbf{A})$ in the phase space $\Gamma$, let us define scalar fields $\psi$ and $\chi$ on $\Delta$ as follows:

i) $\mathcal{L}_{\ell} \psi \hat{=}(\ell \cdot \omega) \widehat{=} \kappa_{(\ell)}$ and $\mathcal{L}_{\ell} \chi \widehat{=}(\ell \cdot \mathbf{A}) \widehat{=}-\Phi_{(\ell)}$; and

ii) $\psi$ and $\chi$ vanish on $S^{-}$, the intersection of $M^{-}$with $\Delta$.ป

Note that these conditions associate with each point of $\Gamma$ a unique pair $(\psi, \chi)$ on $\Delta$ and in the 'extremal' case $\kappa_{(\ell)}=0, \psi$ vanishes identically.

We wish to use the standard procedure [22 involving second variations of the action to define the symplectic structure. [ Let us recall the main steps of this procedure. One first constructs the symplectic current $J$ : Given a point $\gamma$ in the phase space $\Gamma$ and two tangent

${ }^{7}$ Condition ii) serves only to fix the freedom to add constants to $\psi$ and $\chi$. One could envisage replacing it by a different condition. Our results will be insensitive to this choice.

${ }^{8}$ Actually this procedure provides a pre-symplectic structure, i.e., a closed 2-form on the phasespace which, however, is generally degenerate. The vectors in its kernel represent infinitesimal 'gauge transformations'. The physical phase space is obtained by quotienting the space of solutions by gauge transformations and inherits a true symplectic structure from the pre-symplectic structure on the space of solutions. The 2 -form $\Omega$ introduced below is indeed degenerate. However, for simplicity, we will abuse the notation somewhat and refer to $\Omega$ as the symplectic structure. 
vectors $\delta_{1}$ and $\delta_{2}$ at that point, $J$ provides a closed 3 -form $J\left(\gamma ; \delta_{1}, \delta_{2}\right)$ on $\mathcal{M}$. Integrating $d J$ over the part $\tilde{\mathcal{M}}$ of space-time under consideration, one obtains

$$
0=\int_{\tilde{\mathcal{M}}} d J\left(\gamma ; \delta_{1}, \delta_{2}\right)=\oint_{\partial \tilde{\mathcal{M}}} J
$$

Now, if there is no internal boundary, one can choose $\tilde{\mathcal{M}}$ to be a region bounded by any two Cauchy surfaces $M_{1}$ and $M_{2}$ so that the boundary is given by $\partial \tilde{\mathcal{M}}=M_{1} \cup M_{2} \cup \tau_{\infty}$, where $\tau_{\infty}$ is the time-like 'cylinder at infinity'. In simple cases, the asymptotic conditions ensure that the integral $\int_{\tau_{\infty}} J\left(\gamma ; \delta_{1}, \delta_{2}\right)$ vanishes. Then, taking orientations into account, it follows that $\int_{M} J\left(\gamma ; \delta_{1}, \delta_{2}\right)$ is independent of the choice of Cauchy surface $M$. One then sets the symplectic structure to be

$$
\left.\Omega\right|_{\gamma}\left(\delta_{1}, \delta_{2}\right)=\int_{M} J\left(\gamma ; \delta_{1}, \delta_{2}\right)
$$

In our case, the second variation of the action ([II.1) yields the following symplectic current:

$$
J\left(\gamma ; \delta_{1}, \delta_{2}\right)=\frac{-1}{16 \pi G}\left[\delta_{1} \Sigma^{I J} \wedge \delta_{2} A_{I J}-\delta_{2} \Sigma^{I J} \wedge \delta_{1} A_{I J}\right]-\frac{1}{4 \pi}\left[\delta_{1}{ }^{\star} \mathbf{F} \wedge \delta_{2} \mathbf{A}-\delta_{2}{ }^{\star} \mathbf{F} \wedge \delta_{1} \mathbf{A}\right]
$$

Using the fact that the fields $\gamma \equiv(e, A, \mathbf{A})$ satisfy the field equations and the tangent vectors $\delta_{1}, \delta_{2}$ satisfy the linearized equations off $\gamma$, one can directly verify that $J\left(\gamma ; \delta_{1}, \delta_{2}\right)$ is in fact closed as guaranteed by the general procedure involving second variations. It is now natural to choose $\tilde{\mathcal{M}}$ to be a part of our space-time $\mathcal{M}$ bounded by two partial Cauchy surfaces $M_{1}, M_{2}$, the time-like cylinder $\tau_{\infty}$ and a part $\tilde{\Delta}$ of the isolated horizon bounded by $M_{1}$ and $M_{2}$. Again, the asymptotic conditions ensure that the integral of $J$ over $\tau_{\infty}$ vanishes. Hence,

$$
\left(\int_{M_{1}}+\int_{M_{2}}+\int_{\tilde{\Delta}}\right) J\left(\gamma ; \delta_{1}, \delta_{2}\right)=0
$$

However, this does not immediately provide us the conserved symplectic structure because the integral of $J$ over $\tilde{\Delta}$ does not vanish in general. Since the isolation of the horizon implies that there are no fluxes of physical quantities across $\Delta$, one might expect that, although non-zero, the integral over $\tilde{\Delta}$ would be 'controllable'. This is indeed the case. Using the forms (III.2) and (III.3) of $\sum$ and $\underset{\leftarrow}{A}$ on $\Delta$ and the definitions of the potentials $\psi$ and $\chi$, it is easy to verify that the pull-back of the symplectic current to $\Delta$ is itself exact:

$$
\underset{\leftarrow}{J}\left(\gamma ; \delta_{1}, \delta_{2}\right) \widehat{=} d j\left(\gamma, \delta_{1}, \delta_{2}\right)
$$

where the 2 -form $j$ on $\Delta$ is given by:

$$
j\left(\gamma, \delta_{1}, \delta_{2}\right)=\frac{1}{8 \pi G}\left[\delta_{1} \psi \delta_{2}\left({ }^{2} \epsilon\right)-\delta_{2} \psi \delta_{1}\left({ }^{2} \epsilon\right)\right]+\frac{1}{4 \pi}\left[\delta_{1} \chi \delta_{2}{ }^{\star} \mathbf{F}-\delta_{2} \chi \delta_{1}{ }^{\star} \mathbf{F}\right] .
$$

Hence, if $M_{1}$ and $M_{2}$ intersect $\tilde{\Delta}$ in 2-spheres $S_{1}$ and $S_{2}$ respectively, we have: 


$$
\int_{\tilde{\Delta}} J\left(\gamma ; \delta_{1}, \delta_{2}\right)=-\left(\int_{S_{1}}+\int_{S_{2}}\right) j\left(\gamma ; \delta_{1}, \delta_{2}\right)
$$

The negative sign appearing in the above expression is due to the choice of orientation of $S_{\Delta}$, which is induced from $M$ rather than from $\Delta$. Using these results we can define the symplectic structure as:

$$
\begin{aligned}
& \left.\Omega\right|_{\gamma}\left(\delta_{1}, \delta_{2}\right)= \\
& \frac{-1}{16 \pi G} \int_{M}\left[\delta_{1} \Sigma^{I J} \wedge \delta_{2} A_{I J}-\delta_{2} \Sigma^{I J} \wedge \delta_{1} A_{I J}\right]+\frac{1}{8 \pi G} \oint_{S}\left[\delta_{1}\left({ }^{2} \epsilon\right) \delta_{2} \psi-\delta_{2}\left({ }^{2} \epsilon\right) \delta_{1} \psi\right] \\
& -\frac{1}{4 \pi} \int_{M}\left[\delta_{1}{ }^{\star} \mathbf{F} \wedge \delta_{2} \mathbf{A}-\delta_{2}{ }^{\star} \mathbf{F} \wedge \delta_{1} \mathbf{A}\right]+\frac{1}{4 \pi} \oint_{S}\left[\delta_{1}{ }^{\star} \mathbf{F} \delta_{2} \chi-\delta_{2}{ }^{\star} \mathbf{F} \delta_{1} \chi\right]
\end{aligned}
$$

Again, using field equations one can directly verify that the right side of (IV.2) is independent of the choice of the partial Cauchy surface; the symplectic structure is 'conserved'. We will use $(\Gamma, \Omega)$ as our covariant phase space.

Note that, even though the action did not contain a surface term at the horizon, the symplectic structure does. So, the overall situation is the same as in the undistorted, non-rotating case considered in [8]. Finally, our discussion of the action principle and our construction of the covariant phase space is applicable to all weakly isolated horizons $\Delta$; nowhere did we have to restrict ourselves to the non-rotating case.

\section{HAMILTONIAN EVOLUTION AND THE FIRST LAW}

To discuss the first law, we must first define horizon energy, which in turn requires a time evolution field $t^{a}$ on $\mathcal{M}$. Given a vector field $t^{a}$ satisfying appropriate boundary conditions, $\delta_{t}:=\left(\mathcal{L}_{t} e, \mathcal{L}_{t} A, \mathcal{L}_{t} \mathbf{A}\right)$ satisfies the linearized equations for any $\gamma:=(e, A, \mathbf{A})$ in $\Gamma$ and thus defines a vector field on $\Gamma$. 9 This $\delta_{t}$ can be interpreted as the infinitesimal generator of time evolution on the covariant phase space. It is then natural to ask if this vector field is a phase space symmetry, i.e., if $\mathcal{L}_{\delta_{t}} \Omega$ vanishes everywhere on $\Gamma$. The necessary and sufficient condition for this to happen is that there exist a function $H_{t}$ - the Hamiltonian generating the $t$-evolution- such that

$$
\delta H_{t}=\Omega\left(\delta, \delta_{t}\right)
$$

for all vector fields $\delta$ to $\Gamma$. On general grounds, one expects $H_{t}$ to contain a surface term $E_{\mathrm{ADM}}^{t}$ at infinity representing the total (i.e. ADM) energy, and a surface term $E_{\Delta}^{t}$ at the horizon which can be interpreted as the horizon energy, both tied to the evolution field $t^{a}$.

\footnotetext{
${ }^{9}$ In the Lie-derivatives, the internal indices are treated as scalars; thus $\mathcal{L}_{t} e_{a}^{I}=t^{b} \partial_{b} e_{a}^{I}+e_{b}^{I} \partial_{a} t^{b}$. To make $\delta_{t}$ a well-defined vector field on $\Gamma$, we now exclude $M^{ \pm}$from $\mathcal{M}$ and let $\mathcal{M}$ and $\Delta$ be without future and past boundaries. Whenever needed, these boundaries, $M^{ \pm}$and $S^{ \pm}$, can be added by taking the obvious closure of $\mathcal{M}$.
} 
A key question then is to specify the appropriate boundary conditions on $t^{a}$. It is clear that, at infinity, $t^{a}$ should be an asymptotic time-translation, i.e., should approach a timetranslation Killing field of the flat metric used to specify the boundary conditions. At the horizon, on the other hand, the metric is not universal and the space-time defined by a generic point $\gamma$ of the covariant phase space does not admit any Killing field near $\Delta$. Therefore, specification of the boundary conditions at $\Delta$ is not as straightforward as that at infinity. It is for this reason that we now assume that $(\Delta,[\ell])$ is a non-rotating, weakly isolated horizon for all points $\gamma \equiv(e, A, \mathbf{A})$ of the phase space. The problem of specifying the appropriate boundary conditions on $t^{a}$ in the rotating case is more complicated. However, it has been addressed successfully and will be discussed in [13].

Recall that the internal boundary $\Delta$ of $\mathcal{M}$ is equipped with a specific equivalence class $[\ell]$ of vector fields. As discussed in Section [I], these $\ell$ are the isolated horizon analogs of constant multiples of Killing fields on the Killing horizons in static space-times. Therefore, in the non-rotating case, it is natural to demand that, on $\Delta$, the evolution vector field $t^{a}$ should belong to the equivalence class $[\ell]$. This automatically ensures that $\left(\mathcal{L}_{t} e, \mathcal{L}_{t} A, \mathcal{L}_{t} \mathbf{A}\right)$ satisfy the appropriate boundary conditions to define a tangent vector at each point of the phase space $\Gamma$. However, unlike at infinity, the geometry at the horizon is not fixed once and for all. Therefore, it is natural to allow the precise value of the evolution vector field $t^{a}$ on $\Delta$ to vary from one point of the phase space to another. In more familiar terms, this corresponds to allowing the (boundary values of) lapse and shift fields to depend on dynamical fields $(e, A, \mathbf{A})$ themselves, a procedure routinely used in numerical relativity and gauge-fixed calculations in canonical gravity. Following the current terminology in numerical relativity, we will refer to such $t^{a}$ as live evolution vector fields. The use of live fields turns out to be necessary to ensure that $\delta_{t}$ is a phase space symmetry, i.e., yields a Hamiltonian evolution on $(\Gamma, \Omega)$.

Let us fix a live $t^{a}$ whose restriction to the horizon belongs to the equivalence class $[\ell]$ at all points of the phase space. To analyze if $\delta_{t}$ is a Hamiltonian vector field, it is simplest to compute the 1 -form $X_{t}$ on $\Gamma$ defined by

$$
X_{t}(\delta)=\Omega\left(\delta, \delta_{t}\right)
$$

Now $\delta_{t}$ is Hamiltonian - i.e., $\mathcal{L}_{\delta_{t}} \Omega=0$ on $\Gamma$ - if and only if $X_{t}$ is closed, i.e.,

$$
\mathbb{d} \mathrm{X}_{\mathrm{t}}=0
$$

where $\mathbb{d}$ denotes the exterior derivative on (the infinite dimensional) phase space $\Gamma$. If this is the case then, up to an additive constant, the Hamiltonian is given by

$$
\mathbb{d} \mathrm{H}_{\mathrm{t}}=\mathrm{X}_{\mathrm{t}} \text {. }
$$

To calculate the right side of $(\overline{\mathrm{V} .2})$, it is useful to note the following identities from differential geometry:

$$
\begin{array}{rlrl}
\mathcal{L}_{t} A & =t \cdot F+D(t \cdot A) & \mathcal{L}_{t} \Sigma & =t \cdot D \Sigma+D(t \cdot \Sigma)-[(t \cdot A), \Sigma] \\
\mathcal{L}_{t} \mathbf{A}=t \cdot \mathbf{F}+d(t \cdot \mathbf{A}) & \mathcal{L}_{t}{ }^{\star} \mathbf{F} & =t \cdot\left(d^{\star} \mathbf{F}\right)+d\left(t \cdot{ }^{\star} \mathbf{F}\right)
\end{array}
$$


Using these, the field equations satisfied by $(e, A, \mathbf{A})$ and the linearized field equations for $\delta$, we obtain the required expression of $X_{t}$ :

$$
\begin{aligned}
X_{t}(\delta) & :=\Omega\left(\delta, \mathcal{L}_{t}\right) \\
& =\frac{-1}{16 \pi G} \int_{\partial M} \operatorname{Tr}[(t \cdot A) \delta \Sigma-(t \cdot \Sigma) \wedge \delta A]-\frac{1}{4 \pi} \int_{\partial M}(t \cdot \mathbf{A}) \delta\left({ }^{\star} \mathbf{F}\right)-\left(t \cdot{ }^{\star} \mathbf{F}\right) \wedge \delta \mathbf{A} .
\end{aligned}
$$

Note that the expression involves integrals only over the 2-sphere boundaries $S_{\infty}$ and $S_{\Delta}$ of $M$, the partial Cauchy surface used in the evaluation of the symplectic structure; there is no volume term.

The integrals at infinity can be evaluated easily by making use of the fall-off conditions. As one would expect, the electromagnetic term vanishes (because $\mathbf{A}$ falls off at least as $1 / r$ while $\mathbf{F}$ falls off as $1 / r^{2}$ ) while the gravitational term yields precisely the ADM energy $E_{\text {ADM }}^{t}$ associated with the asymptotic time-translation defined by $t^{a}$. At the horizon, we can use equations (III.2) and ([II.3) to show that $t \cdot \Sigma$ contracted on internal indices with $\delta A$ vanishes and (חI.17) implies $t \cdot{ }^{\star} \mathbf{F}=0$ leaving

$$
\begin{aligned}
X_{t}(\delta) & =-\frac{1}{8 \pi G} \int_{S_{\Delta}}(t \cdot \omega) \delta\left({ }^{2} \epsilon\right)-\frac{1}{4 \pi} \int_{S_{\Delta}}(t \cdot \mathbf{A}) \delta\left({ }^{\star} \mathbf{F}\right)+\delta E_{\mathrm{ADM}}^{t} \\
& =-\frac{1}{8 \pi G} \kappa_{(t)} \delta a_{\Delta}-\Phi_{(t)} \delta Q_{\Delta}+\delta E_{\mathrm{ADM}}^{t}
\end{aligned}
$$

where, in the last step, we have used the fact that both $t \cdot \omega=\kappa_{(t)}$ and $t \cdot \mathbf{A}=-\Phi_{(t)}$ are constant on the horizon and the definition ([I.19) of electric charge.

The necessary and sufficient condition for the existence of a Hamiltonian is that $X_{t}$ be closed. Clearly, this is equivalent to

$$
\frac{1}{8 \pi G} \mathbb{d} \kappa_{(\mathrm{t})} \mathbb{A} \mathbb{d} \mathrm{a}_{\Delta}+\mathbb{d} \Phi_{(\mathrm{t})} \mathbb{A} \mathbb{d} \mathrm{Q}_{\Delta}=0
$$

where/Mdenotes the antisymmetric tensor product on $\Gamma$. Now, (V.7) trivially implies that the surface gravity $\kappa_{(t)}$ and the electric potential $\Phi_{(t)}$ at the horizon defined by $t^{a}$ can depend only upon the area and charge of the horizon. Other factors, such as the 'shape' of the distorted horizon, can not affect the values of $\kappa_{(t)}$ or $\Phi_{(t)}$. Finally, ( $\overline{\text {.7.7 }}$ is the necessary and sufficient condition that there exists a function $E_{\Delta}^{t}$, also only of $a_{\Delta}$ and $Q_{\Delta}$ such that

$$
\delta E_{\Delta}^{t}=\frac{1}{8 \pi G} \kappa_{(t)} \delta a_{\Delta}+\Phi_{(t)} \delta Q_{\Delta} .
$$

Since $E_{\Delta}^{t}$ is a function only of $a_{\Delta}$ and $Q_{\Delta}$, it is a function of fields defined locally at the horizon. As noted before, it is natural to interpret $E_{\Delta}^{t}$ as the horizon energy defined by the time translation $t^{a}$. The total Hamiltonian is given by:

$$
H_{t}=E_{\mathrm{ADM}}^{t}-E_{\Delta}^{t} .
$$

Let us summarize. $\mathrm{Eq}(\mathbb{\nabla . 8})$ is a necessary and sufficient condition for the 1 -form $X_{t}$ to be closed. Therefore, the vector field $\delta_{t}$ on $\Gamma$ defined by the space-time evolution field $t^{a}$ is 
Hamiltonian if and only if the first law $(\overline{V .8})$ holds. Thus $(\overline{V .8})$ is a restriction on the choice of the live vector field $t^{a}$ : While any $t^{a}$ (which preserves the boundary conditions) defines an evolution flow on the phase space, it is only when

$$
\frac{1}{8 \pi G} \kappa_{(t)} \mathbb{d a}_{\Delta}+\Phi_{(\mathrm{t})} \mathrm{d} \mathrm{Q}_{\Delta}
$$

is an exact 1-form on $\Gamma$ that this flow is Hamiltonian (i.e., preserves the symplectic structure). At first, this restriction seems somewhat surprising because, in absence of internal boundaries, every vector field $t^{a}$ (which tends to a fixed Killing field of the flat metric at infinity) defines a Hamiltonian evolution. However, even in this context, there is no a priori reason to expect this tight correspondence to hold if one allows general, live vector fields $t^{a}$ whose boundary values at infinity can change from one space-time to another. Finally, we will see in Section VII that every space-time belonging to the phase space $\Gamma$ admits an infinite family of vector fields $t^{a}$ for which $X_{t}$ is closed. Therefore, in particular, the first law does not restrict the 'background' space-times (or the variations $\delta$ ) in any way. Indeed, for any space-time in our phase space, there is an infinite family of first laws, one associated with each permissible $t^{a}$.

We will conclude this section with a few remarks.

i) Form of $H_{t}$ : The Hamiltonian (V.9) contains only surface terms. This may seem surprising because, in the canonical framework, the familiar Hamiltonian contains a volume integral consisting of a linear combination of constraints. While the volume term vanishes 'on shell' and does not contribute to numerical value of the canonical Hamiltonian on physical states, it is nonetheless crucial for obtaining the correct evolution equations since derivatives of the Hamiltonian transverse to the constraint surface are needed to construct the Hamiltonian vector field. The covariant phase space, by contrast, consists only of solutions to the field equations whence the issue of taking 'off shell' derivatives never arises. In diffeomorphism invariant theories, the Hamiltonian on the covariant phase space is always made of surface terms. If space-time has several asymptotic regions, the boundary term in each region defines the standard energy corresponding to that region. Therefore, in the present case, it was natural to interpret $E_{\Delta}^{t}$ as the horizon-energy defined by the $t^{a}$ evolution. Finally, we should emphasize that we used a covariant phase space only for simplicity. The final results go through (and, in fact, were first obtained) in a canonical framework as well.

ii) Comparisons: As noted in the Introduction, all treatments of the first law for nonrotating but possibly distorted horizons available in the literature refer to static space-times. The isolated horizon framework, by contrast, does not refer to a Killing field at all and thus allows a significantly larger class of physically interesting situations. On the other hand,

\footnotetext{
${ }^{10}$ In particular, therefore, Hamiltonians generating diffeomorphism which have support away from the boundaries vanish identically. Unlike their counterparts on the canonical phase space, the infinitesimal phase-space motions induced by such space-time vector fields are in the kernel of the covariant symplectic structure.
} 
since it relies on a Hamiltonian framework, we cannot incorporate phenomenological matter if it does not admit a phase space description. Other treatments based on Hamiltonian methods generally restrict themselves to static space-times with a non-zero surface gravity. This assumption is essential there because those treatments use 'bifurcate' surfaces in an important way and these do not exist in the extremal static solutions where the surface gravity vanishes. In contrast, the results of this section, do not refer to a bifurcate surface and go through irrespective of whether $\kappa_{(t)}$ is non-zero or zero. In a realistic collapse, the physical space-time is not expected to have the bifurcate surface. The present analysis uses only the portion of the physical space-time in which the horizon has settled down with no further in-going radiation, rather than an analytical continuation of the near horizon geometry used in certain approaches. Finally, in contrast to other treatments, we have an infinite family of first laws, one for each evolution field $t^{a}$ for which $\delta_{t}$ is a phase space symmetry.

iii) Non-uniqueness of energy: Each permissible, live $t^{a}$ defines a horizon energy $E_{\Delta}^{t}$. At first it seems surprising that there is so much freedom in the notion of energy. Let us compare the situation at $\mathcal{I}^{+}$, which, like $\Delta$, is null. There, we only have a 3-parameter freedom which, furthermore, can be eliminated simply by fixing a rest frame. How does this difference arise? Recall that energy is (the numerical value of) the generator of an unit time translation. At infinity, all 4-metrics in the phase space approach the same flat metric. Hence, we can simply fix a unit time-translation Killing field $t_{o}^{a}$ of that flat metric near infinity and use its restriction to $\mathcal{I}^{+}$as the unit time-translation for all metrics in the phase space. By contrast, there is no fixed 4-metric near $\Delta$ to which all the metrics in our phase space approach. Hence, we do not have the analog of $t_{o}^{a}$; only the equivalence class $[\ell]$ is now common to all the metrics. If, for a given metric $\tilde{g}_{a b}$ in our collection, we select the time translation represented by a specific $\tilde{\ell}^{a}$ in $[\ell]$, a priori we do not know which vector field $l^{a}$ in $[\ell]$ would represent the 'same' time-translation for another geometry $g_{a b}$. One might imagine using the seemingly simplest strategy: just fix a $\ell_{o}^{a}$ in $[\ell]$ and demand that $t^{a}$ approach that $\ell_{0}^{a}$ for all points $\gamma$ in the phase space. Unfortunately, the strategy is not viable because such a $t^{a}$ fails to define a Hamiltonian evolution in $\Gamma$. space-times, we can overcome this difficulty by always working with the Killing field which is unit at infinity. However, in absence of global Killing fields, the behavior of the evolution vector field $t^{a}$ near the horizon is unrelated to its behavior near infinity. Nonetheless, as we shall show in Section VI1, if one has sufficient control on the space of static solutions of the theory under consideration, it is possible to select a preferred energy function on the phase space and use it as the mass of the isolated horizon. In the Einstein-Maxwell case, all static solutions with horizons are explicitly known whence the strategy is viable.

\footnotetext{
${ }^{11}$ As we saw above, a necessary condition for $\delta_{t}$ to be a Hamiltonian vector field on $\Gamma$ is that $\kappa_{(t)}$ is a function only of $a_{\Delta}$ and $Q_{\Delta}$. Therefore, if we can find a tangent vector $\delta$ in the phase space with $\delta a_{\Delta}=\delta Q_{\Delta}=0$ but $\delta \kappa_{(t)} \neq 0, t^{a}$ can not define a Hamiltonian evolution. It is easy to find such a tangent vector $\delta$ for this $t^{a}$.
} 


\section{YANG-MILLS FIELD}

In the previous three sections we restricted our attention to Einstein-Maxwell theory. We will now indicate how Yang-Mills fields can be included. This section is divided into three parts. In the first, we discuss restrictions on the Yang-Mills fields due to the horizon boundary conditions and introduce the notion of a 'Yang-Mills gauge adapted to the horizon'. In the second part, we discuss the action principle and construct the covariant phase space for Einstein-Yang-Mills theory. Using this formalism, in the third subsection, we introduce a Hamiltonian generating time evolution and extend the first law to the Yang-Mills case.

\section{A. Preliminaries}

We will restrict ourselves to compact gauge groups $G$ and Yang-Mills connections defined on trivial bundles. Since the bundle is trivial, the connection gives rise to a smooth, globally defined Lie algebra valued 1-form, $\mathbf{A}$. As usual, the Yang-Mills derivative operator $\mathbf{D}$ will be defined as $\mathbf{D} \lambda=\partial \lambda+[\mathbf{A}, \lambda]$, where $\partial$ is a flat Yang-Mills connection, and the field strength, $\mathbf{F}$, via

$$
\mathbf{F}:=d \mathbf{A}+\mathbf{A} \wedge \mathbf{A} .
$$

The stress energy tensor, $\mathbf{T}$, is given in terms of the field strength as

$$
\mathbf{T}_{a b}=\frac{1}{4 \pi}\left[\mathbf{F}_{a c}{ }^{i} \mathbf{F}_{b}{ }^{c}{ }_{i}-\frac{1}{4} g_{a b} \mathbf{F}_{c d}{ }^{i} \mathbf{F}^{c d}{ }_{i}\right],
$$

where the label $i$ runs over the internal indices in the Lie algebra of the group G.

Let us begin by examining how the isolated horizon boundary conditions restrict the form of the field strength, $\mathbf{F}$, on $\Delta$. Since the Yang-Mills stress energy tensor has the same form as the Maxwell one, ([I.14), the analysis is completely analogous to that of Section

IIT. Therefore, we shall not include derivations of the results, but instead highlight the differences.

Recall that, on a non-expanding horizon, $R_{a b} \ell^{a} \ell^{b} \hat{=0}$ whence $T_{a b} \ell^{a} \ell^{b} \hat{=} 0$. This has several consequences for the Yang-Mills field. In particular, one concludes

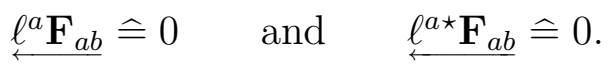

These two restrictions guarantee there is no flux of Yang-Mills field across the horizon. Making use of the specific form of the stress energy tensor, we also conclude

$$
\Phi_{02}=\frac{1}{2} R_{a b} m^{a} m^{b} \hat{=} 0 \quad \text { and } \quad \Phi_{20}=\frac{1}{2} R_{a b} \bar{m}^{a} \bar{m}^{b} \hat{=} 0 .
$$

Our next task is to define the Yang-Mills equivalents of the electric and magnetic charges of the horizon. Naively, one might consider integrating $\mathbf{F}$ and ${ }^{\star} \mathbf{F}$ over a 2 -sphere cross section 
of the horizon as was done in the Maxwell theory. However, these 2-forms now have a free internal index and are only gauge covariant rather than gauge invariant. Since there is no preferred internal basis at the horizon, the integrals would fail to be well-defined. Therefore, we must look for 2-forms which are gauge invariant. A natural quantity to consider is the norm of $\mathbf{F}$, defined by the Killing-Cartan form $K_{i j}$ on the Lie-algebra of $G$ and the (contravariant) ${ }^{2} \epsilon$ on the horizon:

$$
|\mathbf{F}|:=\left[\left({ }^{2} \epsilon \cdot \mathbf{F}\right)^{i}\left({ }^{2} \epsilon \cdot \mathbf{F}\right)^{j} K_{i j}\right]^{\frac{1}{2}}
$$

(Although the contravariant ${ }^{2} \epsilon$ is ambiguous up to terms of the type $\ell^{[a} V^{b]}$ where $V^{a}$ is any vector field tangential to $\Delta$, this ambiguity does not affect $|\mathbf{F}|$ because of (VI.3). The norm of ${ }^{\star} \mathbf{F}$ is defined analogously. These two quantities are gauge invariant and allow us to define the electric and magnetic Yang-Mills charges of the horizon:

$$
Q_{\Delta}^{Y M}:=-\frac{1}{4 \pi} \oint_{S_{\Delta}}\left|{ }^{\star} \mathbf{F}\right|^{2} \epsilon \quad \quad \quad \text { and } \quad P_{\Delta}^{Y M}:=-\frac{1}{4 \pi} \oint_{S_{\Delta}}|\mathbf{F}|^{2} \epsilon
$$

Recall that the unusual signs in the definitions of the charges arise due to the orientation of the $S_{\Delta}$ — the normal to the two sphere is inward pointing. In Maxwell theory, the magnetic charge is zero unless we consider either connections on non-trivial bundles or allow 'wire singularities'. As is well known, this is not true for Yang-Mills theory: the magnetic charge can be non-zero even if we restrict attention to smooth fields on a trivial bundle.

We would now like to verify that the charges defined in (VI.6) are independent of the cross section of the horizon $S_{\Delta}$ on which the integration is performed. The isolated horizon boundary conditions guarantee this is the case. First, recall the geometric identity

$$
\mathcal{L}_{\ell} \underset{\leftarrow}{\mathbf{F}} \hat{=} \ell \cdot \underset{\mathrm{DF}}{\longleftarrow}-[(\ell \cdot \mathbf{A}), \underset{\leftarrow}{\mathbf{F}}]+\underset{\mathbf{D}(\ell \cdot \mathbf{F})}{ } .
$$

A similar expression for ${ }^{\star} \mathbf{F}$ is also true. The first term on the right hand side vanishes due to the field equations and the third term is zero due to the previous restriction on $\mathbf{F}$, (VI.3). Therefore at the isolated horizon,

$$
\mathcal{L}_{\ell} \mathbf{F}=-[(\ell \cdot \mathbf{A}), \mathbf{F}] \quad \text { and } \quad \mathcal{L}_{\ell_{\longleftarrow}^{\star}} \mathbf{F}=-\left[(\ell \cdot \mathbf{A}),{ }^{\star} \mathbf{F}\right] .
$$

In the Maxwell case, $\mathbf{F}$ and ${ }^{\star} \mathbf{F}$ are Lie dragged by $\ell$. However, for non-Abelian fields, this is not a gauge invariant statement; the terms on the right hand sides of (VI.8) are necessary for gauge invariance. Although the field strength and its dual are not Lie dragged along $\ell^{a}$, recalling that $\mathcal{L}_{\ell}^{2} \epsilon \widehat{=} 0$ and using the cyclic property of the trace, it is straightforward to demonstrate that their norms are:

$$
\mathcal{L}_{\ell}|\mathbf{F}| \widehat{=} 0 \quad \text { and }\left.\quad \mathcal{L}_{\ell}\right|^{\star} \mathbf{F} \mid \widehat{=} 0
$$

This result, along with $(\mathbb{\nabla 1 . 3})$, guarantees that the charges $Q_{\Delta}^{Y M}$ and $P_{\Delta}^{Y M}$ are independent of the choice of cross section $S_{\Delta}$ of the horizon. 
Let us consider the remaining components of the Yang-Mills field. The boundary conditions place no restrictions on $\mathbf{F}_{a b} n^{a} m^{b}$ and ${ }^{\star} \mathbf{F}_{a b} n^{a} m^{b}$ at all. As in the electromagnetic case, these components describe the radiation flowing along the horizon. The isolated horizon boundary conditions allow radiation arbitrarily close to - and even at - the horizon, provided none crosses it.

We have so far restricted our attention to the field strength and its dual. However, in the action principle and phase space, the basic variable will be the Yang-Mills connection A. Let us begin with the definition of the Yang-Mills equivalent of the electric potential. Recall that, given an $\ell$, the electric potential was defined in Section $\mathbb{I I G}$ as $\Phi_{(\ell)} \widehat{=}-(\ell \cdot \mathbf{A})$. This definition is not appropriate in the Yang-Mills case since the resulting potential has a free internal index and is therefore not gauge invariant. Instead, we define the Yang-Mills potential, $\Phi_{(\ell)}^{\mathrm{YM}}$ to be negative the norm of $(\ell \cdot \mathbf{A})$ :

$$
\Phi_{(\ell)}^{\mathrm{YM}}: \hat{=}-|(\ell \cdot \mathbf{A})|
$$

This gives us a gauge invariant potential at the horizon.

As in Maxwell theory, we need to constrain the form of $\mathbf{A}$ at the horizon. Several considerations motivate our choice of these boundary conditions. First they must be chosen so that the action principle is well defined. Second, if the gauge group is U(1) the boundary conditions should reduce to those given in Section $\amalg \mathrm{CO}$ for the electromagnetic field. Finally, we should be able to show that the Yang-Mills electric potential is constant on the horizon. These considerations suggest the following definition:

Definition 6: The connection $\mathbf{A}$ will be said to be in a gauge adapted to the isolated horizon $(\Delta,[\ell])$ if it satisfies the following two conditions

- (i) The Yang-Mills potential is constant on the horizon

$$
d \Phi_{(\ell)}^{\mathrm{YM}} \widehat{=} 0
$$

- (ii) The dual of the field strength $\left({ }^{\star} \mathbf{F}\right)$ and $(\ell \cdot \mathbf{A})$ point in the same Lie algebra direction,

$$
(\ell \cdot \mathbf{A})^{i} \propto\left({ }^{2} \epsilon \cdot{ }^{\star} \mathbf{F}\right)^{i}
$$

on the horizon.

These boundary conditions satisfy the requirements discussed above. First, it is straightforward to show that in the $\mathrm{U}(1)$ case, condition (i) is equivalent to requiring $\mathcal{L}_{\ell} \mathbf{A} \widehat{=} 0$ and (ii) is redundant. Second, as we shall see in Section VIB, these boundary conditions are also sufficient to make the variational principle well defined.

It is not difficult to show that these conditions can always be satisfied. The remaining gauge freedom is simply $\mathbf{A} \rightarrow g^{-1} \mathbf{A} g+g^{-1} \partial g$, where $g$ satisfies $\mathcal{L}_{\ell} g \widehat{=} 0$. 


\section{B. Action and Phase Space}

In this section we will consider the first order action for Einstein-Yang-Mills theory on the manifold $\mathcal{M}$ described in Section IIIB. The basic fields will consist of the triplet $\left(e_{a}^{I}, A_{a I}{ }^{J}, \mathbf{A}_{a}^{i}\right)$, where $e_{a}^{I}$ and $A_{a I}^{J}$ are the tetrad and Lorentz connection and $\mathbf{A}_{a}^{i}$ is the YangMills connection. The gravitational fields, $e_{a}^{I}$ and $A_{a I}{ }^{J}$ satisfy the same boundary conditions (at $\Delta$ and infinity) as in Section एIIB. Furthermore, we require the Yang-Mills fields to be in a gauge adapted to the horizon and assume they fall off sufficiently fast at infinity. ${ }^{2}$ The Einstein-Yang-Mills action is

$$
S(e, A, \mathbf{A})=\frac{-1}{16 \pi G} \int_{\mathcal{M}} \Sigma^{I J} \wedge F_{I J}+\frac{1}{16 \pi G} \int_{\tau_{\infty}} \Sigma^{I J} \wedge A_{I J}-\frac{1}{8 \pi} \int_{\mathcal{M}} \operatorname{Tr}\left[\mathbf{F} \wedge{ }^{\star} \mathbf{F}\right] .
$$

The gravitational part of the action has previously been discussed in Section [II], therefore we shall only consider in detail the Yang-Mills terms and verify the variational principle is well defined. Taking into account the results of Section [II], a variation of the action can be expressed as

$$
\delta S(e, A, \mathbf{A})=\int_{\mathcal{M}} \text { Equations of Motion } \cdot \delta \phi-\frac{1}{4 \pi} \int_{\Delta} \operatorname{Tr}\left[\delta \mathbf{A} \wedge{ }^{\star} \mathbf{F}\right] .
$$

We must demonstrate the boundary term at $\Delta$ vanishes due to the conditions imposed on the Yang-Mills fields. Using (V1.3) and (V1.12), one can show that the trace in (V1.14) can be replaced by a product of norms:

$$
\operatorname{Tr}\left[\delta \mathbf{A} \wedge{ }^{\star} \mathbf{F}\right]=\left(\delta \Phi_{(\ell)}^{\mathrm{YM}}-\mathrm{c}_{\delta} \Phi_{(\ell)}^{\mathrm{YM}}\right)\left|{ }^{\star} \mathbf{F}\right|^{\Delta_{\epsilon}},
$$

where ${ }^{\Delta} \epsilon=n \wedge^{2} \epsilon$ is the volume form on $\Delta$ and $\delta \ell=c_{\delta} \ell$.

In the action principle, variations are performed keeping data fixed at the initial and final slices. In particular $\delta \Phi_{(\ell)}^{\mathrm{YM}}$ and $c_{\delta}$ vanish there. However, the boundary conditions guarantee that $\Phi_{(\ell)}^{\mathrm{YM}}$, and hence its variation, is constant on $\Delta$. Since, $\delta \Phi_{(\ell)}^{\mathrm{YM}}$ and $c_{\delta}$ vanish on the initial cross section of the horizon and are constant, it follows that $\delta \Phi_{(\ell)}^{\mathrm{YM}} \hat{=} 0$ and $c_{\delta} \widehat{=} 0$. Therefore, the Yang-Mills horizon boundary term vanishes; the action principle is well defined in the presence of Yang-Mills fields. As in the Einstein-Maxwell case, boundary conditions played a crucial role in demonstrating the viability of the action.

\footnotetext{
${ }^{12}$ More specifically we require the fall off conditions on the Yang-Mills connection to be such that all integrals, in particular the symplectic structure, are finite and yet the asymptotic electric and magnetic charges are not forced to vanish. While it is not trivial to meet these conditions (for examples the conditions used in [23,12] appear not to lead to a well-defined symplectic structure) they can be met. However, as in the rest of the paper, for brevity, we will not spell out the boundary conditions at infinity in detail.
} 
We now wish to construct the covariant phase space and symplectic structure. As before, points in covariant phase space $\Gamma$ will consist of histories which satisfy the EinsteinYang-Mills field equations, appropriate falloff conditions at infinity and the isolated horizon boundary conditions at $\Delta$. Before proceeding further, we shall once again need to introduce an additional field at the horizon. This can be regarded as 'potential' for the Yang-Mills potential $\Phi^{\mathrm{YM}}$. Given any point $(e, A, \mathbf{A})$ in the phase space $\Gamma$, let us define the scalar field $\chi$ on $\Delta$ as follows: i) $\mathcal{L}_{\ell} \chi=-\Phi_{(\ell)}^{\mathrm{YM}}$; and ii) $\chi$ vanishes on $S^{-}$, the intersection of $M^{-}$with $\Delta$. These conditions are identical to those imposed in the Maxwell case.

Once again, we take second variations of the action in order to obtain a symplectic structure. Since the gravitational terms are exactly the same in Section [II], we shall only describe in detail the Yang-Mills part of the symplectic structure. The second variation of the action (V1.13) yields the following symplectic current:

$$
J\left(\gamma ; \delta_{1}, \delta_{2}\right)=J_{\text {grav }}-\frac{1}{4 \pi} \operatorname{Tr}\left[\delta_{1}^{\star} \mathbf{F} \wedge \delta_{2} \mathbf{A}-\delta_{2}{ }^{\star} \mathbf{F} \wedge \delta_{1} \mathbf{A}\right] .
$$

Using the fact that the field equations and linearized field equations are satisfied, one can directly verify that $J\left(\gamma ; \delta_{1}, \delta_{2}\right)$ is indeed a closed 3 -form. We again choose the spacetime region of interest, $\tilde{\mathcal{M}}$, to be that part of the spacetime $\mathcal{M}$ bounded by $M_{1}, M_{2}$, infinity and a portion $\tilde{\Delta}$ of the isolated horizon. Integrating $d J$ over $\tilde{\mathcal{M}}$ and using asymptotic falloff conditions, we obtain

$$
\left(\int_{M_{1}}+\int_{M_{2}}+\int_{\tilde{\Delta}}\right) J\left(\gamma ; \delta q_{1}, \delta_{2}\right)=0
$$

The integral of $J$ over $\tilde{\Delta}$ does not vanish but, as in Section III, the pull back of $J$ to $\Delta$ is exact. Therefore, we can express the integral over $\tilde{\Delta}$ of $J$ as integrals over the initial and final 2-spheres $S_{1}$ and $S_{2}$. Using these results, and keeping track of orientations, we obtain the symplectic structure:

$$
\begin{aligned}
& \left.\Omega\right|_{\gamma}\left(\delta_{1}, \delta_{2}\right)= \\
& -\frac{1}{16 \pi G} \int_{M}\left[\delta_{1} \Sigma^{I J} \wedge \delta_{2} A_{I J}-\delta_{2} \Sigma^{I J} \wedge \delta_{1} A_{I J}\right]+\frac{1}{8 \pi G} \oint_{S}\left[\delta_{1}{ }^{2} \epsilon \delta_{2} \psi-\delta_{2}{ }^{2} \epsilon \delta_{1} \psi\right] \\
& -\frac{1}{4 \pi} \int_{M}\left[\delta_{1}{ }^{\star} \mathbf{F} \wedge \delta_{2} \mathbf{A}-\delta_{2}{ }^{\star} \mathbf{F} \wedge \delta_{1} \mathbf{A}\right]+\frac{1}{4 \pi} \oint_{S}\left[\delta_{1}\left(\left|{ }^{\star} \mathbf{F}\right|{ }^{2} \epsilon\right) \delta_{2} \chi-\delta_{2}\left(\left|{ }^{\star} \mathbf{F}\right|{ }^{2} \epsilon\right) \delta_{1} \chi\right] .
\end{aligned}
$$

Full use of the isolated horizon boundary conditions has been made in obtaining this symplectic structure. In particular, to obtain the given form of the Yang-Mills surface term, we have used the fact that $(\ell \cdot \mathbf{A})$ and $\left({ }^{2} \epsilon \cdot{ }^{\star} \mathbf{F}\right)$ point in the same direction in the Lie algebra. Using field equations one can directly verify that the right side of (VI.16) is independent of the choice of the partial Cauchy surface; the symplectic structure is 'conserved'. We will use $(\Gamma, \Omega)$ as our covariant phase space. 


\section{Hamiltonian and First Law}

In this subsection we will generalize the arguments of Section $\square$ to obtain an expression for the energy of the horizon in Einstein-Yang-Mills theory. To do so, we must specify a time evolution vector field $t^{a}$. As before, we require $t^{a}$ to be a member of the preferred equivalence class $\left[\ell^{a}\right]$ at the horizon (this again requires restriction to non-rotating isolated horizons) and approach unit time translation asymptotically. Given $t^{a}$ we can calculate the infinitesimal generator of time evolution, $\delta_{t}=\left(\mathcal{L}_{t} e, \mathcal{L}_{t} A, \mathcal{L}_{t} \mathbf{A}\right)$, and determine whether it is Hamiltonian. Recall that $\delta_{t}$ is Hamiltonian if and only if the 1-form $X_{t}$ on the phase space defined by

$$
X_{t}(\delta):=\Omega\left(\delta, \delta_{t}\right)
$$

is closed. Let us calculate $X_{t}$. The gravitational part will be identical to the expression obtained in Section Q , therefore we shall concentrate on the Yang-Mills terms. As with the Maxwell field, the Lie derivatives of the Yang-Mills fields can be re-expressed using the following identities:

$$
\mathcal{L}_{t} \mathbf{A}=t \cdot \mathbf{F}+\mathbf{D}(t \cdot \mathbf{A}) \quad \mathcal{L}_{t}{ }^{\star} \mathbf{F}=t \cdot\left(\mathbf{D}^{\star} \mathbf{F}\right)-\left[\ell \cdot \mathbf{A},{ }^{\star} \mathbf{F}\right]+\mathbf{D}\left(t \cdot{ }^{\star} \mathbf{F}\right) .
$$

Making use of these expressions, the field equations satisfied by $(e, A, \mathbf{A})$ and the linearized field equations for $\delta$, we obtain the required expression for $X_{t}$ :

$$
X_{t}(\delta)=\frac{-1}{16 \pi G} \int_{\partial M}(t \cdot A) \delta \Sigma-(t \cdot \Sigma) \wedge \delta A-\frac{1}{4 \pi} \int_{\partial M} \operatorname{Tr}\left[(\mathrm{t} \cdot \mathbf{A}) \delta\left({ }^{\star} \mathbf{F}\right)-\left(\mathrm{t} \cdot{ }^{\star} \mathbf{F}\right) \wedge \delta \mathbf{A}\right] .
$$

As before the expression involves integrals only over the 2 -sphere boundaries $S_{\infty}$ and $S_{\Delta}$ of $M$; there is no volume term. The gravitational terms yield $\delta E_{\mathrm{ADM}}^{t}$ at infinity and $-(1 / 8 \pi G) \kappa_{(t)} \delta a_{\Delta}$ at the horizon. The Yang Mills term at infinity vanishes due to falloff conditions, therefore we need only calculate the Yang-Mills contribution at the horizon. This is composed of two terms, the second of which vanishes due to the restriction (VI.3) which guarantees $\ell \cdot{ }^{\star} \mathbf{F}=0$. Since we are in a gauge adapted to the horizon, $(\ell \cdot \mathbf{A})$ and ${ }^{\star} \mathbf{F}$ point in the same internal direction. This allows us to replace the trace in the first term by norms:

$$
\operatorname{Tr}\left[(\mathrm{t} \cdot \mathbf{A}) \delta\left({ }^{\star} \mathbf{F}\right)\right]=-\Phi_{(\mathrm{t})}^{\mathrm{YM}} \delta\left(\left|{ }^{\star} \mathbf{F}\right|{ }^{2} \epsilon\right)
$$

and guarantees that $\Phi_{(t)}^{\mathrm{YM}}$ is constant on the horizon. Making use of the definition of YangMills electric charge, (V1.6), we obtain

$$
X_{t}(\delta)=-\frac{1}{8 \pi G} \kappa_{(t)} \delta a_{\Delta}-\Phi_{(t)}^{\mathrm{YM}} \delta Q_{\Delta}^{\mathrm{YM}}+\delta E_{\mathrm{ADM}}^{t} .
$$

Recall that the necessary and sufficient condition for the existence of a Hamiltonian is that $X_{t}$ be closed. Clearly, this is equivalent to 


$$
\frac{1}{8 \pi G} \mathbb{d} \kappa_{(\mathrm{t})} \wedge \mathbb{d} \mathrm{a}_{\Delta}+\mathbb{d} \Phi_{(\mathrm{t})}^{\mathrm{YM}} \wedge \mathbb{d} \mathrm{Q}_{\Delta}^{\mathrm{YM}}=0,
$$

Once again, we conclude that the surface gravity $\kappa_{(t)}$ and the Yang-Mills potential $\Phi_{(t)}^{\mathrm{YM}}$ at the horizon defined by $t^{a}$ can depend only upon the area $a_{\Delta}$ and charge $Q_{\Delta}^{\mathrm{YM}}$ of the horizon. Finally, (VI.20) is also the necessary and sufficient condition that there exist a function $E_{\Delta}^{t}$, also only of $a_{\Delta}$ and $Q_{\Delta}^{\mathrm{YM}}$ such that

$$
\delta E_{\Delta}^{t}=\frac{1}{8 \pi G} \kappa_{(t)} \delta a_{\Delta}+\Phi_{(t)}^{\mathrm{YM}} \delta Q_{\Delta}^{\mathrm{YM}} .
$$

As before, $E_{\Delta}^{t}$ is interpreted as the horizon energy defined by the time translation $t^{a}$. We conclude that the vector field $\delta_{t}$ is Hamiltonian if and only if the first law, (VI.21), holds.

We will conclude this section with a few remarks.

i) The derivation of the first law and its final form are completely analogous to those in the Einstein-Maxwell theory. By contrast, in the discussion of the first law for undistorted isolated horizons of [12], certain restrictions were imposed on the permissible variations $\delta$ in the Einstein-Yang-Mills case. In our treatment, subtleties arise only in the definition of a canonical horizon mass (see Section VIIC) rather than the discussion of the first law itself.

ii) Although the Yang-Mills magnetic charge $P_{\Delta}^{\mathrm{YM}}$ will generically not be zero, no term involving $\delta P_{\Delta}^{\mathrm{YM}}$ arises in the first law.

iii) How does our result compare with those previously available [23,24]? In [24] the first law for Yang-Mills fields is proved for globally stationary spacetimes and small perturbations from one such space-time to another. Assuming the Yang-Mills fields fall off sufficiently fast at infinity, (in the non-rotating case) the first law of [24] then reads

$$
\delta M=\frac{1}{8 \pi G} \kappa \delta a+\int_{\text {Hor }} \operatorname{Tr}\left[\phi \delta^{\star} \mathrm{F}\right] .
$$

Here, $M$ is the ADM mass evaluated at infinity, while all terms on the right hand side are evaluated at the horizon. Due to a different gauge choice at the horizon, the authors define a Lie algebra valued potential $\phi$ and leave the ' $\Phi \delta Q$ ' term inside an integral. However, the general form of this first law is the same as ours. In this sense, our framework generalizes the results of [24] to non-static contexts.

In [23], the first law is proved for globally stationary spacetimes and arbitrary small departures therefrom. However, there are a number of important differences between these results and the ones obtained in this paper. In the non-rotating case, the first law of [23] reads

$$
\delta M_{\mathrm{ADM}}+V \delta Q=\frac{1}{8 \pi G} \kappa \delta a
$$

where $V$ and $Q$ are the Yang-Mills potential and charge evaluated at infinity while $\kappa$ and $a$ are of course evaluated at the horizon. Because of the non-Abelian nature of the Yang-Mills field, unlike in the Maxwell case, the charge $Q$ evaluated at infinity is now quite different 
from the charge evaluated at the horizon and, as in the Maxwell theory, the potential $V$ evaluated at infinity has no direct bearing on the potential at the horizon. Furthermore, that calculation makes an essential use of the bifurcation 2-sphere and all fields are required to be smooth there. This restriction implies that the Yang-Mills potential at the horizon vanishes. (The same is true if one restricts the analysis of [23] to the Maxwell case.)

The first law derived in the isolated horizon framework is valid also in presence of radiation in the exterior space-time region and makes no reference to the bifurcation 2-sphere. (Although we restricted ourselves to the non-rotating case, rotation has been incorporated in this framework in 13.). Furthermore, it has the aesthetically pleasing feature that all quantities that appear in (VI.21) - including the energy $E_{\Delta}^{t}$, the potential $\Phi_{(t)}^{\mathrm{YM}}$ and the charge $Q_{\Delta}^{\mathrm{YM}}$ - are evaluated at the horizon. In particular, one can now meaningfully consider the physical process version in which one does an experiment at the horizon by dropping a test particle/field and changing the horizon charge infinitesimally. More generally (V1.21) is genuinely a law governing the mechanics of the horizon.

\section{HORIZON MASS}

For notational simplicity, we will say that a (live) vector field $t^{a}$ is permissible if it gives rise to a Hamiltonian evolution. We saw in Sections $\square$ and $\nabla \mathbb{1}$ that each permissible vector field $t^{a}$ defines a horizon energy $E_{\Delta}^{t}$. In the phase space framework, $E_{\Delta}^{t}$ has a direct interpretation: it is the surface term at the horizon in the expression of the Hamiltonian generating the $t^{a}$-evolution. However, in many physical applications - such as the study of black hole mergers - one is interested in properties of a specific space-time, rather than the full phase space. Then, it is useful to have at one's disposal a canonical notion of energy, the analog of the ADM energy in the rest frame at infinity. This quantity could then be interpreted as the horizon mass. In this section, we will introduce this notion in detail. The discussion is divided into three parts. In the first, we consider the Einstein-Maxwell theory; in the second, we discuss dilatonic couplings [9]; and, in the third, we analyze the Einstein-Yang-Mills system.

\section{A. Einstein-Maxwell theory}

In Section $\nabla$ we showed that $t^{a}$ is permissible if and only if $(\nabla .7)$ holds on the phase space. We will now construct a large family of permissible evolution fields $t^{a}$. Fix any regular function $\kappa_{o}$ of two variables $a_{\Delta}$ and $Q_{\Delta}$. Then, given any point $\gamma \equiv(e, A, \mathbf{A})$ of $\Gamma$, we define (the boundary value of) the vector field $t^{a}$ as follows. Consider the vector field $\ell^{a}$ on $\Delta$ defined by the tetrad, $\ell^{a}=e_{I}^{a} \ell^{I}$, and denote by $\kappa_{(\ell)}$ the surface gravity associated with it. Then, $\kappa_{o}=c \kappa_{(\ell)}$ for some constant $c$. Let us set $t^{a}=c \ell^{a}$. Repeating this procedure at each phase-space point $\gamma$, we obtain a live vector field $t^{a}$ with $\kappa_{(t)}=\kappa_{o}$. (The resulting $c$ will be constant on $\Delta$ but a function on the phase space.) Next, consider the electro-magnetic potential, which is guaranteed to be constant on $\Delta$ by our boundary conditions but whose 
value at any phase space point is so far completely free. We will now use (V.7) to fix it. Equation (V.7) implies:

$$
\frac{\partial \kappa_{(t)}}{\partial Q_{\Delta}}=\frac{\partial \Phi_{(t)}}{\partial a_{\Delta}} .
$$

Since $\kappa_{(t)}=\kappa_{o}$ is known, we can simply integrate the equation for $\Phi_{(t)}$ as a function of $a_{\Delta}$ and $Q_{\Delta}$. Furthermore, the solution is unique if we impose the physical condition that $\Phi_{(t)}$ should vanish whenever $Q_{\Delta}=0$. Thus, starting from any regular function $\kappa_{o}$ of $a_{\Delta}$ and $Q_{\Delta}$, we have obtained a permissible evolution field $t^{a}$. Conversely, it is easy to verify that every permissible vector field arises via this construction. There is clearly a very large family of such live vector fields.

An obvious question is if there is a 'canonical' or 'natural' choice of $t^{a}$ ? We will now show that the answer is in the affirmative. Recall that, in the Einstein-Maxwell theory, there is precisely a 2-parameter family of globally static solutions admitting horizons: the Reissner-Nordström family. (Since $\mathbf{A}$ is required to be a globally defined connection on a trivial $U(1)$ bundle, the magnetic charge is zero on the entire phase space.) Let us focus on this family. Denote by $\xi^{a}$ the static Killing field which is unit at infinity. Its surface gravity is a specific function of $a_{\Delta}$ and $Q_{\Delta}$ :

$$
\kappa_{(\xi)}=\frac{1}{2 R_{\Delta}}\left(1-\frac{G Q_{\Delta}^{2}}{R_{\Delta}^{2}}\right) .
$$

As before, $R_{\Delta}$ is the horizon radius, defined by $a_{\Delta}=4 \pi R_{\Delta}^{2}$. We can therefore use $\kappa_{(\xi)}$ in place of $\kappa_{o}$ in the above construction. The resulting permissible, live vector field $t_{o}^{a}$ agrees with $\xi^{a}$ on the horizon of every static solution. This property is satisfied only if we set $\kappa_{o}=\kappa_{(\xi)}$.

Next, we can 'integrate' ( $\nabla .8)$ to obtain the horizon energy $E_{\Delta}^{t_{o}}$. Although a priori there is the freedom to add a constant, we can fix it by requiring that the energy vanish as $a_{\Delta}$ and $Q_{\Delta}$ tend to zero. Indeed, we have no choice in this since one cannot construct a quantity with dimensions of mass from the fundamental constants that appear in the Einstein-Maxwell theory. (Einstein-Yang-Mills theory does admit such a constant and we will see in Section VII that it leads to an interesting modification of the situation discussed here.) Let us define the horizon mass via

$$
M_{\Delta}=E_{\Delta}^{t_{o}}
$$

To justify this definition, let us begin by restricting to static solutions. In each static solution, we are free to extend $t_{o}^{a}$ such that it coincides with the Killing field $\xi^{a}$. General symplectic arguments imply that, on any connected component of the space of static solutions, the numerical value of the total Hamiltonian, generating evolution along $\xi^{a}$, must be constant (see, e.g., [8].) In the Einstein-Maxwell case, there is a single connected component and, by the dimensional argument given above, the numerical value of the Hamiltonian must vanish on it. Hence, from (V.9) it follows that, on any static solution, 


$$
H_{t_{o}}=M_{\mathrm{ADM}}-M_{\Delta}=0 .
$$

On a general solution, of course, $M_{\mathrm{ADM}}$ would be greater than $M_{\Delta}$, the difference being equal to the energy in radiation. If the horizon is complete in the future and time-like infinity $i^{+}$ satisfies certain regularity conditions, as in [8] one can argue that the difference is precisely the total energy radiated across $\mathcal{I}^{+}$and hence $M_{\Delta}$ equals the future limit of the Bondi mass. These considerations support our interpretation of $M_{\Delta}$ as the horizon mass.

Finally, since we now have a canonical evolution field $t_{o}^{a}$, we can drop the suffix $t$ on surface gravity and electromagnetic potential and write the first law (V.8) in the more familiar form:

$$
\delta M_{\Delta}=\frac{1}{8 \pi G} \kappa \delta a_{\Delta}+\Phi \delta Q_{\Delta}
$$

In contrast to treatments based on static space-times, the quantities that enter this law are all defined at the horizon. Therefore, as pointed out in [8], it is now possible to interpret this law also in the 'active' sense where one considers physical processes which increase the area and the charge of a given horizon. To our knowledge, the standard proofs of this physical version [5, 10] are not applicable to processes in which the background has non-zero electric charge and the process changes it infinitesimally.

We will conclude with a few remarks.

i) In the above discussion, the permissible evolution field $t_{o}^{a}$ was constructed by setting $t_{o}^{a}=c \ell^{a}$ where $c$ is given by $\kappa_{o} \equiv\left(1 / 2 R_{\Delta}\right)\left(1-G\left(Q_{\Delta} / R_{\Delta}\right)^{2}\right)=c \kappa_{(\ell)}$. For $c$ to be welldefined, it is necessary that $\kappa_{o}$ vanishes whenever $\kappa_{(\ell)}$ does. Therefore, for the mass to be well-defined, we must excise those points from the phase space at which $\kappa_{(\ell)}$ vanishes but $\kappa_{o}$ does not. However, this is not a serious limitation. In particular, we still retain all static solutions including the extremal ones at which $\kappa_{o}$ vanishes.

ii) Since we have a specific $\kappa_{o}$, we can use (V.7) to obtain the corresponding electrostatic potential: $\Phi_{o}=Q_{\Delta} / R_{\Delta}$. Furthermore, by integrating (V.8) it is easy to express $M_{\Delta}$ explicitly in terms of the horizon parameters:

$$
M_{\Delta}=\frac{1}{4 \pi G} \kappa a_{\Delta}+\Phi Q_{\Delta}=\frac{R_{\Delta}}{2 G}\left(1+\frac{G Q_{\Delta}^{2}}{R_{\Delta}^{2}}\right) .
$$

Thus, the functional dependence of $M_{\Delta}$ on the horizon parameters at any point of the phase space is the same as in static space-times. Note that this is a result of the framework, not an assumption. Its derivation involved two distinct steps. First, and most importantly, the first law (D.8) arose as a necessary and sufficient condition for the existence of a consistent Hamiltonian framework. Second, the freedom in $t^{a}$ was exploited in order to construct the preferred, permissible evolution field $t_{o}^{a}$. It is quite significant that $M_{\Delta}$ can be expressed so simply using just the parameters defined locally at the horizon even when there is radiation arbitrarily close to it. This fact is likely to play an important role in the problem of extracting physics in the strong field regimes from numerical simulations of black hole collisions [18]. It is important to notice that although we made use of our knowledge of static solutions to arrive at a canonical $t_{o}^{a}$ and the mass function $M_{\Delta}$, the final result (VII.1) makes no reference 
to these solutions. $M_{\Delta}$ is a simple function of the parameters which can be directly computed from the geometry of any one isolated horizon.

iii) In the earlier work [8] on undistorted horizons, one restricted oneself to the preferred evolution field $t_{o}^{a}$ from the very beginning (although this vector field was selected using a different but equivalent procedure) The a priori freedom in the choice of a permissible $t^{a}$ was not discussed and the first law appeared only in the more familiar form, given above.

\section{B. Dilatonic coupling}

The Einstein-Maxwell-dilaton system was studied in some detail in the undistorted case in [9]. We will revisit it here in the more general context considered in this paper because it brings out a subtlety in the definition of the horizon mass $M_{\Delta}$ and the associated first law.

The dilaton is a scalar field $\phi$ which can couple to the Maxwell field in a non-standard fashion. The coupling is governed by a constant $\alpha$. If $\alpha=0$, one obtains the standard Einstein-Maxwell-Klein-Gordon theory and the situation then is completely analogous to the Einstein-Maxwell theory considered above. If $\alpha=1$, the theory represents the low energy limit of string theory. In this case, there are some interesting differences from the EinsteinMaxwell theory considered in this paper. To bring out these differences, in this subsection we will set $\alpha=1$. (The situation for a general value of $\alpha$ is discussed in [9] where one can also find details on the material summarized below.)

In the standard formulation, the theory has three charges, all defined at infinity; the ADM mass $M_{\mathrm{ADM}}$, the usual electric charge $Q_{\infty}$ and another charge $\tilde{Q}_{\infty}$ :

$$
Q_{\infty}=\frac{1}{4 \pi} \oint_{S_{\infty}}{ }^{\star} \mathbf{F} \quad \text { and } \quad \tilde{Q}_{\infty}=\frac{1}{4 \pi} \oint_{S_{\infty}} e^{-2 \phi \star} \mathbf{F}
$$

$\tilde{Q}$ is conserved in space-time (i.e. its value does not change if the 2 -sphere of integration is deformed) while $Q$ is not. From the perspective of the isolated horizons, it is more useful to use $a_{\Delta}, Q_{\Delta}, \tilde{Q}_{\Delta}$ as the basic chargestu:

\footnotetext{
${ }^{13}$ This strategy seems to have generated a misunderstanding (see, e.g., [29]) that the first law was obtained in 8,9.12] merely by identifying the parameters labeling a general isolated horizon with those of static horizons and then using the Smarr formulas available in the static context. This was not the case. Rather, static solutions were used only to select the appropriate normalization of the evolution vector field $t^{a}$ at the horizon. The Hamiltonian framework was then used to define the horizon mass without any reference to Smarr formulas. As in this section, the mass was then shown to reproduce the Smarr-type formulas on general horizons.

${ }^{14}$ In the undistorted case, the dilaton is constant on $\Delta$ and hence we can replace $Q_{\Delta}$ by $\phi_{\Delta}$ as in [9].
} 


$$
Q_{\Delta}=\frac{1}{4 \pi} \oint_{S_{\Delta}}{ }^{\star} \mathbf{F}, \quad \text { and } \quad \tilde{Q}_{\Delta}=\frac{1}{4 \pi} \oint_{S_{\Delta}} e^{-2 \phi \star} \mathbf{F} .
$$

Although the standard electric charge is not conserved in space-time, it is conserved along $\Delta$ whence $Q_{\Delta}$ is well-defined.

It is straightforward to extend the construction of the phase space to include the dilaton. The only difference is that the charge $Q$ in equations $(\nabla .6)-(\nabla .8)$ is replaced by $\tilde{Q}$. With this minor change, the discussion of the first part of Section VIIA is also unaffected. Thus, given any function $\kappa_{o}$ of $a_{\Delta}$ and $\tilde{Q}_{\Delta}$, we can construct a permissible, (live) evolution field $t^{a}$.

The difference arises in the next step where we constructed a preferred $t_{o}^{a}$. With the dilatonic coupling, the theory has a unique [25] three parameter family of static solutions which can be labeled by $\left(a_{\Delta}, Q_{\Delta}, \tilde{Q}_{\Delta}\right)$. As in the Reissner Nordström family, these solutions are spherically symmetric. In terms of these parameters, the surface gravity $\kappa_{(\xi)}$ of the static Killing field which is unit at infinity is given by:

$$
\kappa_{(\xi)}=\frac{1}{2 R_{\Delta}}\left[1+2 G \frac{Q_{\Delta} \tilde{Q}_{\Delta}}{R_{\Delta}^{2}}\right]\left[1-2 G \frac{Q_{\Delta} \tilde{Q}_{\Delta}}{R_{\Delta}^{2}}\right]^{-\frac{1}{2}} .
$$

The problem in the construction of the preferred $t_{o}^{a}$ is that we need a function $\kappa_{o}$ which depends only on $a_{\Delta}$ and $\tilde{Q}_{\Delta}$. Therefore, we can no longer set $\kappa_{o}=\kappa_{(\xi)}$ on the entire phase space because $\kappa_{(\xi)}$ depends on all three horizon parameters.

To extract the mass function $M_{\Delta}$ on the phase space, we can proceed as follows. Let us foliate $\Gamma$ by $Q_{\Delta}=$ const surfaces. On each leaf, $\kappa_{(\xi)}$ trivially depends only on $a_{\Delta}$ and $\tilde{Q}_{\Delta}$ and so we can set $\kappa_{o}=\kappa_{(\xi)}$. Therefore, by the procedure outlined in Section VIIA, we obtain a (live) vector field $t_{o}^{a}$ and can define the mass $M_{\Delta}(\gamma)=E_{\Delta}^{t_{o}}(\gamma)$ for all points $\gamma$ on this leaf. Repeating this procedure for each leaf, we obtain a live vector field $t_{o}^{a}$ and a mass function $M_{\Delta}$ everywhere on $\Gamma$. However, the surface gravity $\kappa_{\left(t_{o}\right)}$ now depends on all three parameters, rather than just $a_{\Delta}$ and $\tilde{Q}_{\Delta}$. Therefore, the first law (V.8) cannot hold for arbitrary variations $\delta$ and consequently $\delta_{t_{o}}$ fails to be a Hamiltonian vector field. Put differently, although there is a multitude of permissible, live vector fields, each leading to a first law, none of them can coincide with the Killing field $\xi^{a}$ (which is unit at infinity) on all static solutions. This is a significant departure from the Einstein-Maxwell case considered above.

Nonetheless, (modulo the caveat discussed in the first remark at the end of Section VIIA) the above procedure does provide us with a well-defined mass function $M_{\Delta}$ on the entire phase space which can be expressed in terms of the horizon parameters as

$$
M_{\Delta}=\frac{R_{\Delta}}{2 G}\left[1-2 G \frac{Q_{\Delta} \tilde{Q}_{\Delta}}{R_{\Delta}^{2}}\right]^{-\frac{1}{2}} .
$$

It equals $M_{\mathrm{ADM}}$ in static space-times and has other properties which motivated our interpretation of $M_{\Delta}$ as the horizon mass in the Einstein-Maxwell case. Since this function is well-defined on the entire phase space, we can simply vary it and express the result in terms of the horizon parameters. The result is: 


$$
\delta M_{\Delta}=\frac{1}{8 \pi G} \kappa \delta a_{\Delta}+\hat{\Phi} \delta \hat{Q}_{\Delta}
$$

where $\kappa=\kappa_{\left(t_{o}\right)}, \hat{\Phi}^{2}=\left(Q_{\Delta} \tilde{Q}_{\Delta} / R_{\Delta}^{2}\right)$ and $\hat{Q}_{\Delta}^{2}=Q_{\Delta} \tilde{Q}_{\Delta}$. Thus, although there is still a first law in terms of $t_{o}^{a}$ and $M_{\Delta}$, it does not have the canonical form (V.8) because $t_{o}^{a}$ is not a permissible vector field. More generally, in theories with multiple scalar fields [26], if one focuses only on static sectors, one obtains similar 'non-standard' forms of the first law with work terms involving scalar fields. This reflects the fact that there is no permissible vector field $t^{a}$, defined for all points of the phase space, which coincides with the properly normalized Killing field on all static solutions. In the undistorted case, the analysis was carried out only in terms of the vector field $t_{o}^{a}$ and the horizon mass $M_{\Delta}$ [9]. The resulting first law had the above form.

Alternatively, one can restrict oneself to variations $\bar{\delta}$ which are tangential to the leaves of the phase space foliation constructed above. Since $t_{o}^{a}$ is a permissible vector field for any one leaf, we obtain the standard first law

$$
\bar{\delta} M_{\Delta}=(1 / 8 \pi G) \kappa_{\left(t_{o}\right)} \bar{\delta} a_{\Delta}+\Phi_{\left(t_{o}\right)} \bar{\delta} \tilde{Q}_{\Delta}
$$

for the restricted variations. The idea of using such restricted variations was suggested in [12] in the context of Yang-Mills fields (although the foliations and other details were not spelled out there).

To summarize, because there is now a three parameter family of static solutions rather than two - or, more precisely, because the standard surface gravity $\kappa_{(\xi)}$ in static space-times depends on $a_{\Delta}, \tilde{Q}_{\Delta}$ and $Q_{\Delta}$ - a canonical, permissible evolution field is no longer available. However, there is still a multitude of permissible evolution fields and corresponding first laws. Furthermore, one can still define a canonical mass function $M_{\Delta}$ on the entire phase space.

\section{Yang-Mills fields}

In Einstein-Maxwell theory, with and without the dilaton, there is no way to construct a quantity with the dimensions of mass from the fundamental constants in the theory. The situation is different for Einstein-Yang-Mills theory because the coupling constant $g$ has dimensions $(L M)^{-1 / 2}$. The existence of a quantity with units of mass has interesting consequences which we will now discuss.

Let us begin with a summary of the known static solutions in Yang-Mills theory. First, the Reissner-Nordström family constitutes a continuous two parameter set of static solutions of the Einstein-Yang-Mills theory, labelled by $\left(a_{\Delta}, Q_{\Delta}^{\mathrm{YM}}\right)$. In addition, there is a 1-parameter family of 'embedded Abelian solutions' with (a fixed) magnetic charge $P_{\Delta}^{o}$, labelled by $\left(a_{\Delta}, P_{\Delta}^{o}\right)$. Finally, there are families of 'genuinely non-Abelian solutions'. For these, the analog of the Israel theorem for Einstein-Maxwell theory fails to hold [27]; the theory admits static solutions which need not be spherically symmetric. In particular, an infinite family of 
solutions labelled by two integers $\left(n_{1}, n_{2}\right)$ is known to exist. All static, spherically symmetric solutions are known and they correspond to the infinite sub-family $\left(n_{1}, n_{2}=0\right)$, labelled by a single integer. However, the two parameter family is obtained using a specific ansätz, so there may well exist other static solutions. Although the available information on static solutions is quite rich, in contrast to the Einstein-Maxwell-Dilaton system, one is still rather far from having complete control of the static sector of the Einstein-Yang-Mills theory.

The zeroth and first laws do hold in the Einstein-Yang-Mills case. At present, however, we can only hope to repeat the strategy used in the last two sub-sections to define a canonical mass function $M_{\Delta}$ on portions of the phase space. In order to define it on the full phase space, of the 'uniqueness' and 'completeness' conjectures of [12] will have to hold (possibly with a suitable modification. I. Nevertheless, new insight into the static solutions can be obtained by restricting attention to certain leaves of the phase space. The basic idea is taken from [12] but applied in a slightly different manner to the more general context of distorted horizons.

Consider a connected component of the known static solutions, labelled by $\vec{n} \equiv\left(n_{1}, n_{2}\right)$. This is a 1-dimensional sub-space of the phase space which we denote $S_{\vec{n}}$. Each point in $S_{\vec{n}}$ can be labelled by the value of the horizon area $a_{\Delta}$. Calculate the surface gravity $\kappa_{(\xi)}$ for this family, where $\xi^{a}$ is the static Killing field which it unit at infinity and set $\kappa_{o}=\kappa_{(\xi)}$ in the construction sketched in Section VIIA. We then obtain a live vector field $t_{o}^{a}$, and the corresponding first law

$$
\delta E_{\Delta}^{t_{o}}=\frac{1}{8 \pi G} \kappa_{\left(t_{o}\right)} \delta a_{\Delta}
$$

on the full phase space.

When restricted to $S_{\vec{n}}$, we can interpret $E_{\Delta}^{t_{o}}$ as the horizon mass $M_{\Delta}^{(\vec{n})}$ and replace $\kappa_{\left(t_{o}\right)}$ by the function $\beta_{\vec{n}}\left(a_{\Delta}\right)$ used in the literature: $\beta_{\vec{n}}=2 \kappa_{\left(t_{o}\right)} R_{\Delta}$. Then, by integrating the first law along $S_{\vec{n}}$, one obtains:

$$
M_{\Delta}^{(\vec{n})}=\frac{1}{2 G} \int_{0}^{R_{\Delta}} \beta_{\vec{n}}(x) d x
$$

where, we have used the fact that, since $E_{\Delta}^{\left(t_{o}\right)}$ is a surface integral at $\Delta$, it vanishes as the horizon area goes to zero. Thus, the horizon mass is completely determined by $\beta_{\vec{n}}\left(a_{\Delta}\right)$.

Next, we use the fact that the Hamiltonian given by $H_{t_{o}}=E_{\mathrm{ADM}}^{t_{o}}-E_{\Delta}^{t_{o}}($ see $(\overline{\nabla .9}))$ is constant on each connected, static sector if $t_{o}^{a}$ coincides with the static Killing field on the entire sector. By construction, our $t_{o}^{a}$ has this property. In the Einstein-Maxwell case, since

\footnotetext{
${ }^{15}$ For example it may be appropriate to restrict oneself to the class of space-times admitting isolated horizons which are complete in the future. Physically, this is the most interesting case since such horizons would represent the asymptotic geometry resulting from a gravitational collapse or black hole mergers.
} 
there is no constant with the dimension of energy, it follows that the restriction of $H_{\mathrm{ADM}}^{t_{o}}$ to the static sector must vanish. The situation is quite different in Einstein-Yang-Mills theory where the Yang-Mills coupling constant $g$ provides a scale. In $c=1$ units, $(g \sqrt{G})^{-1} \sim$ Mass. Therefore, we can only conclude

$$
M_{\mathrm{ADM}}^{(\vec{n})}=M_{\Delta}^{(\vec{n})}+(g \sqrt{G})^{-1} C^{(\vec{n})}
$$

for some $\vec{n}$-dependent constant $C^{(\vec{n})}$. As the horizon radius shrinks to zero, the static solution 27,28 under consideration tends to the solitonic solution with the same 'quantum numbers' $\vec{n}$. Hence, by taking this limit, we conclude $(g \sqrt{G})^{-1} C^{(\vec{n})}=M_{\mathrm{ADM}}^{\text {soliton, }(\overrightarrow{\mathrm{n}})}$. Therefore, we have the following interesting relation between the black hole and solitonic solutions:

$$
M_{\mathrm{ADM}}^{\mathrm{BH},(\vec{n})}=\frac{1}{2 G} \int_{0}^{R_{\Delta}} \beta_{\vec{n}}(x) d x+M_{\mathrm{ADM}}^{\text {soliton, }(\vec{n})}
$$

where the integral of $\beta_{\vec{n}}$ is evaluated on the 1-dimensional 'parameter space' of $S_{\vec{n}}$ (given by the horizon radius). Furthermore, as is clear from the above discussion, the ADM mass of the soliton is a multiple of $(g \sqrt{G})^{-1}$. Thus, somewhat surprisingly, the derivation of the first law in the isolated horizon framework has led to an interesting relation between the ADM masses of black holes and their solitonic analogs in the static sector.

\section{DISCUSSION}

In the first part of this paper, we introduced the notions of weakly isolated and isolated horizons. In contrast with earlier work [8,9, 12, the definitions allow for the possible presence of distortion and rotation at the horizon. In addition, the present definitions are more geometric and intrinsic; in particular, they never refer to a foliation.

The notion of an isolated horizon, unlike that of an event horizon, is completely quasilocal. One can test if a given 3-surface in space-time is (weakly) isolated or not simply by examining space-time geometry at the surface. Furthermore, space-times admitting an isolated horizon $\Delta$ need not admit any Killing field even in a neighborhood of $\Delta$. In particular, they can admit radiation in the exterior region. Therefore, such space-times can serve as more realistic models of late stages of a gravitational collapse or black hole merger. In [18] the near $\Delta$ geometry of vacuum solutions is examined in detail using similar techniques to those used at null infinity. The resulting structure - presence of a preferred 'rest frame', constrains on possible isometries, Bondi-type expansions of the metric — should be useful to extract physics in the strong field regime of general relativity, especially in the problem of binary black hole collisions.

This paper, however, focused on another aspect of isolated horizons: extensions of the zeroth and first laws of black hole mechanics. All previous discussions of these laws were restricted to perturbations of stationary black holes. Using Lagrangian and Hamiltonian frameworks, we extended these laws to arbitrary space-times admitting non-rotating isolated horizons in Einstein-Maxwell-dilaton and Einstein-Yang-Mills theory. Furthermore, 
the analysis suggests that it should be rather easy to incorporate other forms of matter, provided they admit Lagrangian and Hamiltonian descriptions.

The generalization of black hole mechanics presented in this paper has several interesting features. First, all quantities that enter the first laws are defined locally at the horizon $\Delta$. In standard treatments, some quantities such as area and surface gravity are defined at the horizon. Others, like energy and sometimes [23] even the Yang-Mills/Maxwell charge and potential, are evaluated at infinity. In part because of this 'mismatch', to our knowledge the 'physical process version' of the first law [10] had not previously been established for processes which change the charge of the black hole. Since all quantities in the present treatment are defined locally at the horizon, it is now straightforward to establish the law for such processes [8]. Secondly, other treatments based on a Hamiltonian framework [10,32, 23] often critically use the bifurcate 2 -surface which does not exist in the extremal case. Therefore, extremal black holes are often excluded from the first law. The present analysis never makes reference to bifurcate surfaces (which do not exist in physical space-times resulting from gravitational collapse). Therefore, our discussion of the first law holds also in the extremal case. Thirdly, with obvious modifications of boundary conditions at infinity, our analysis includes cosmological horizons where thermodynamic considerations are also applicable [11].

Finally, and perhaps most importantly, our analysis sheds new light on the 'origin' of the first law: it arose as a necessary and sufficient condition for the existence of a Hamiltonian generating time evolution. A new feature of our framework is the existence of an infinite family of first laws corresponding to the infinite family of 'permissible' vector fields $t^{a}$. (A vector field $t^{a}$ is permissible if it is Hamiltonian, that is, induces canonical transformations on the phase space.) In theories where we have sufficient control on the space of static solutions, such as Einstein-Maxwell, one can select a natural evolution field $t_{o}^{a}$. Corresponding to evolution along this $t_{o}^{a}$, there is a canonical notion of energy which can be interpreted as the mass of the isolated horizon. There exist also preferred values of surface gravity and electric potential and a canonical first law. This additional structure is extremely useful in other applications of the framework, such as extraction of physical information from numerical simulations of black-hole collisions. However, it is not essential to the discussion of mechanics: our derivation of the first law in Sections $\nabla$ and $\nabla$ does not require any knowledge of the static sector of the theory.

The Hamiltonian approach to black hole mechanics has appeared in the literature before, most notably in the work of Brown and York [14]. The spirit of the Brown-York approach is similar to ours. In particular, they do not restrict themselves to stationary situations. However, in that work, the focus is on an outer, time-like boundary whereas our focus is on the inner, null boundary representing the isolated horizon. Conserved quantities in presence of internal boundaries were recently discussed also by Julia and Silva [30 in a more general context of theories with gauge symmetries. As in our framework, their treatment exploits the simplifications that occur in a first order formalism and the final surface-integral expressions of conserved charges are dictated by the precise boundary conditions imposed at the internal boundaries. Their treatment is based on superpotentials and is thus complements the Hamiltonian methods used here and in [14. 
In this paper, the Lagrangian and Hamiltonian frameworks are based on real tetrads and Lorentz connections. It is therefore quite straightforward to extend our analysis to any space-time dimension. Indeed, it has already been extended to $2+1$ dimensions in [31]. However, our phase space - and especially the explicit symplectic structure used here - is tailored to the Einstein-matter system. While it should be possible to extend it to higher derivative theories of gravity as in [32], that task would not be as simple.

\section{ACKNOWLEDGMENTS}

We are grateful to Chris Beetle and Jerzy Lewandowski for countless discussions. We thank Alejandro Corichi and Daniel Sudarsky for stimulating correspondence, Jiri Bicak and Werner Israel for pointing out references on distorted black holes and Thibault Damour, Sean Hayward and Bob Wald for helpful comments. This work was supported in part by the NSF grants PHY94-07194, PHY95-14240, INT97-22514 and by the Eberly research funds of Penn State. SF was supported in part by a Braddock Fellowship.

\section{APPENDIX A: EXAMPLES OF DISTORTED HORIZONS}

Because of the no hair theorems in the Einstein-Maxwell theory, distorted horizons have received a rather limited attention in the literature. Therefore, in this appendix we will discuss a few explicit examples in Einstein-Maxwell theory. For a general construction and an existence result, see 18,33.

To obtain explicit solutions, one has to impose symmetries. All solutions considered in this section will be static and axi-symmetric. As one would expect from the no-hair theorems, they fail to be asymptotically flat, whence they fail to represent isolated black holes in the standard sense. Nonetheless, they all satisfy the isolated horizon boundary conditions. That framework also serves to 'explain' the otherwise surprising feature that the surface gravity of these solutions depends only on the area and the charge and is insensitive to the distortion parameters.

In the literature on static, distorted black holes, it is generally assumed that the solution is valid only in a finite region around the horizon and its distant behavior is suitably modified by the far-away matter which causes the distortion. For undistorted isolated horizons, RobinsonTrautman space-times [15] offer interesting examples of vacuum, asymptotically flat solutions which admit isolated horizons but no Killing fields whatsoever. Distorted analogs of these solutions are not known but presumably exist. It would be interesting to find them.

\section{Black Hole in a magnetic universe}

Let us begin with a simple example: an uncharged black hole in an 'external magnetic field' which distorts the horizon. The specific solution we wish to consider is static and 
axisymmetric and was first obtained in the Ernst-potential framework [34. The magnetic field is non-zero on the horizon. Thus, one has to consider the full set of Einstein-Maxwell equations on the horizon.

The space-time has topology $S^{2} \times \mathbb{R}^{2}$ and the metric is given by

$$
d s^{2}=F^{2}\left[-\left(1-\frac{2 M}{r}\right) d t^{2}+\frac{d r^{2}}{1-2 M / r}+r^{2} d \theta^{2}\right]+\frac{r^{2} \sin ^{2} \theta}{F^{2}} d \phi^{2}
$$

where

$$
F=1+\frac{1}{4} B_{0}^{2} r^{2} \sin ^{2} \theta
$$

$B_{0}$ is a constant and on the axis the magnetic field is given by $B=B_{0} d r$. Because $F$ diverges at infinity, the metric fails to be asymptotically flat. For $M=0$, the metric reduces to that of the Melvin universe and for $M \neq 0$ it admits a Killing horizon at $r=2 M$. To examine the behavior at the horizon, let us first cast the metric in the Eddington-Finkelstein coordinates $(v, r, \theta, \phi)$ where $d v=d t+\left(1-\frac{2 M}{r}\right)^{-1} d r$ :

$$
d s^{2}=-F^{2}\left(1-\frac{2 M}{r}\right) d v^{2}+2 F^{2} d v d r+F^{2} r^{2} d \theta^{2}+\frac{r^{2} \sin ^{2} \theta}{F^{2}} d \phi^{2} .
$$

Since the metric is not asymptotically flat, the standard procedure of normalizing the Killing field to be unit at infinity is not applicable. Thus, we only have an equivalence class $[\ell]$ of (preferred) null normals to the horizon, $\ell \propto \frac{\partial}{\partial v}$. Let $\Delta$ be the Killing horizon and assume the Killing field $\frac{\partial}{\partial v}$ is a member of the equivalence class $[\ell]$. It follows trivially that $(\Delta,[\ell])$ is a non-rotating isolated horizon. If $B_{0} \neq 0$, the scalar curvature ${ }^{2} R$ of the horizon 2-metric has $\theta$-dependence; the horizon is distorted. However, an explicit calculation shows that, as in the Schwarzschild space-time, the surface gravity $\kappa$ is given by $1 / 2 r \equiv 1 / 2 R_{\Delta}$ and the electrostatic potential $\Phi$ vanishes on $\Delta$. At first, it is quite surprising that while the presence of distortion affects $\mu, \Phi_{11}, \Psi_{2}$ and ${ }^{2} R$, it does not affect $\kappa$ or $\Phi$. However, as we saw in Section $\mathrm{V}$, this result is to be expected from the general Hamiltonian considerations.

\section{Distorted black holes as special cases of Weyl solutions}

In this sub-section, we will review the construction of a large family of distorted black holes starting from Weyl solutions [35] and a recent generalization of these results to include electric charge [36.

A general class of static, axisymmetric spacetimes was found by Weyl in 1917 [37. The metric for such a spacetime can be cast in the following form:

$$
d s^{2}=-e^{2 \psi} d t^{2}+e^{2(\gamma-\psi)}\left(d \rho^{2}+d z^{2}\right)+e^{-2 \psi} \rho^{2} d \phi^{2}
$$

where $\psi$ and $\gamma$ are smooth functions of $\rho$ and $z$. Einstein's vacuum equations expressed in terms of $\psi$ and $\gamma$ take a particularly simple form. The equation for $\psi$, 


$$
\psi_{, \rho \rho}+\frac{\psi_{, \rho}}{\rho}+\psi_{, z z}=0
$$

is simply the Laplace equation in flat space with cylindrical coordinates $(\rho, z, \phi)$. (In addition, $\psi$ has to be independent of the angular coordinate $\phi$.) Given a solution for $\psi$, the function $\gamma$ can be determined by simple integration:

$$
\begin{aligned}
& \gamma_{, \rho}=\rho\left[\psi_{, \rho}^{2}-\psi_{, z}^{2}\right] \\
& \gamma_{, z}=2 \rho\left[\psi_{, \rho} \psi_{, z}\right]
\end{aligned}
$$

The Schwarzschild metric is of course a particular solution to these equations and corresponds to choosing for $\psi$ and $\gamma$ :

$$
\psi=\psi_{\mathrm{S}}:=\frac{1}{2} \ln \left(\frac{L-M}{L+M}\right) \quad \text { and } \quad \gamma=\gamma_{\mathrm{S}}:=\frac{1}{2} \ln \left(\frac{L^{2}-M^{2}}{L^{2}-\eta^{2}}\right)
$$

where $L=\frac{1}{2}\left(l_{+}+l_{-}\right), \eta=\frac{1}{2}\left(l_{+}-l_{-}\right)$with $l_{+}=\sqrt{\rho^{2}+(z+M)^{2}}$ and $l_{-}=\sqrt{\rho^{2}+(z-M)^{2}}$ and $M$ is the mass of the Schwarzschild solution. Note that $\psi$ is just the Newtonian potential due to a rod of length $2 M$ placed symmetrically about the origin on the z-axis. Both $\psi_{S}$ and $\gamma_{S}$ diverge logarithmically in the limit $\rho \rightarrow 0$ (for $|z| \leq M$ ). In order to recast this solution in the standard Schwarzschild form, one must transform from $(z, \rho)$ to the Schwarzschild coordinates $(r, \theta)$ by

$$
z=(r-M) \cos \theta \quad \text { and } \quad \rho^{2}=r^{2}(1-2 M / r) \sin ^{2} \theta
$$

This coordinate transformation shows that the horizon, $r=2 M$, corresponds to the line segment $\rho=0,|z| \leq M$ in Weyl coordinates. Therefore, the Weyl coordinates cover only the exterior of the horizon.

Now, the key point is that (A.4), the only field equation one has to solve, is linear. Hence we can 'distort' the Schwarzschild solution simply by adding to $\psi_{\mathrm{S}}$ any solution $\psi_{D}$ of the flat space Laplace equation which is regular along the $z$-axis [35]. Thus, we can set

$$
\psi=\psi_{\mathrm{S}}+\psi_{D} \quad \text { and } \quad \gamma=\gamma_{\mathrm{S}}+\gamma_{D}
$$

Substituting these expressions into (A.4) and (A.5) and using the forms of the Schwarzschild functions $\psi_{S}$ and $\gamma_{S}$, one can show that at $\rho=0$,

$$
\left.\left.\gamma_{D}\right|_{\rho=0} \widehat{=} 2 \psi_{D}\right|_{\rho=0} .
$$

This fact plays an important role in analyzing the horizon structure.

In Schwarzschild coordinates, the distorted metric takes the form

$$
d s^{2}=-e^{2 \psi_{D}}(1-2 M / r) d t^{2}+\frac{e^{2\left(\gamma_{D}-\psi_{D}\right)}}{(1-2 M / r)} d r^{2}+e^{2\left(\gamma_{D}-\psi_{D}\right)} r^{2} d \theta^{2}+r^{2} \sin ^{2} \theta e^{-2 \psi_{D}} d \phi^{2}
$$


As usual, the metric has a coordinate singularity at $r=2 M$. Let us therefore introduce the Eddington-Finkelstein coordinate $v$ as before. The metric can be re-expressed in $(v, r, \theta, \phi)$ coordinates as

$$
\begin{gathered}
d s^{2}=-e^{2 \psi_{D}}(1-2 M / r) d v^{2}+(1-2 M / r)^{-1} e^{2 \psi_{D}}\left(e^{2\left(\gamma_{D}-2 \psi_{D}\right)}-1\right) d r^{2} \\
+2 e^{2 \psi_{D}} d v d r+e^{2\left(\gamma_{D}-\psi_{D}\right)} r^{2} d \theta^{2}+e^{-2 \psi_{D}} r^{2} \sin ^{2} \theta d \phi^{2}
\end{gathered}
$$

Using condition (A.9) it is not difficult to show that the coefficient of $d r^{2}$ in the metric is regular at $r=2 M$ [35].

It is immediately obvious from (A.11) that the $r=2 M$ surface is a Killing horizon of $\frac{\partial}{\partial v}$. However, we can not select a preferred normalization for this vector field since the metric is not asymptotically flat. As in the last sub-section, let $\Delta$ be the Killing horizon and choose $\ell \propto \frac{\partial}{\partial v}$. Then, it is straightforward to verify that $(\Delta,[\ell])$ is a complete, non-rotating isolated horizon. Let us calculate the value of surface gravity for $\ell=\frac{\partial}{\partial v}$. We obtain

$$
\kappa \widehat{=}\left(e^{2 \psi_{D}-\gamma_{D}}\right) \frac{1}{2 r} \widehat{=} \frac{1}{2 r}
$$

where we arrived at the last expression by using (A.9). Again, while spin coefficient $\operatorname{Re}[\mu]$, the Weyl component $\Psi_{2}$ and the scalar curvature ${ }^{2} R$ of the horizon metric all depend on the distortion function $\psi_{D}$, somewhat surprisingly the surface gravity $\kappa_{(\ell)}$ does not.

The natural question is whether the above framework can be extended to obtain distorted Reissner-Nordström solutions. This turns out to be non-trivial because the key equation (A.4) now acquires a source term from the electromagnetic field and this field itself depends non-trivially on $\psi$ through the Maxwell equations. At first, the coupled system appears to be hopelessly difficult. However, there exists a prescription 38 for defining a new potential $\tilde{\psi}$ in terms of $\psi$ and the electromagnetic field such that $\tilde{\psi}$ satisfies the flat space Laplacian (A.4). Using this method, the known distorted black hole solutions were recently generalized to the charged case [36]. The distorted Reissner-Nordström solution is given by the metric

$$
\begin{aligned}
d s^{2}= & -\left(1-2 M / r+Q^{2} / r^{2}\right) e^{2 \psi_{D}} d t^{2}+\left(1-2 M / r+Q^{2} / r^{2}\right)^{-1} e^{2\left(\gamma_{D}-\psi_{D}\right)} d r^{2} \\
& +e^{2\left(\gamma_{D}-\psi_{D}\right)} r^{2} d \theta^{2}+e^{-2 \psi_{D}} r^{2} \sin ^{2} \theta d \phi^{2} .
\end{aligned}
$$

The forms of $\psi_{D}$ and $\gamma_{D}$ are now substantially more complicated than in the uncharged case. Nonetheless, it is still possible to show that (A.9) continues to hold. As before this equality implies that the apparent singularity at $r_{H}^{2}-2 M r_{H}+Q^{2}=0$ is only a coordinate singularity. The surface defined by $r=r_{H}$ is a Killing horizon of $\frac{\partial}{\partial t}$. There is once again, no natural way to normalize the Killing field, so we only have an equivalence class $\left[\ell^{a}\right]$ of null normals to the Killing horizon. $(\Delta,[\ell])$ is a non-rotating isolated horizon.

The surface gravity of $\frac{\partial}{\partial t}$ is given by

$$
\kappa=\frac{1}{2 r_{H}}\left(1-\frac{Q^{2}}{r_{H}^{2}}\right) .
$$


Again, the surface gravity is independent of the distortion of the horizon and has the same dependence on the horizon radius $R_{\Delta}$ (which turns out to be equal to $r_{H}$ ) and charge $Q$ as in Reissner-Nordström spacetime. Considerations of Section $\nabla$ suggest this peculiar behavior of $\kappa$ in all these examples is not accidental but can be 'explained' from general Hamiltonian considerations which led us to the first law.

\section{APPENDIX B: THE NEWMAN-PENROSE FORMALISM}

\section{Notation and Conventions}

Let us begin with a summary of the Newman-Penrose formalism (see [39] or 40 42] for a complete account). Apart from the spacetime signature which we take to be $(-,+,+,+)$, we will follow the conventions used in [12]. Consider a tetrad of null vectors $n, \ell, m$ and $\bar{m}$ ( $n$ and $\ell$ are real while $m$ is complex) which satisfy

$$
\begin{aligned}
& n \cdot \ell=-1 \quad n \cdot m=0 \quad n \cdot \bar{m}=0 \\
& \ell . m=0 \quad \ell \cdot \bar{m}=0 \quad m \cdot \bar{m}=1 \text {. }
\end{aligned}
$$

The directional derivatives along the basis vectors are denoted by

$$
D=\ell^{a} \nabla_{a} \quad \Delta=n^{a} \nabla_{a} \quad \delta=m^{a} \nabla_{a} \quad \bar{\delta}=\bar{m}^{a} \nabla_{a} .
$$

The full the information contained in the connection is expressed in terms of twelve complex scalars called the Newman-Penrose spin coefficients defined as follows:

$$
\begin{array}{llll}
\kappa=-m^{a} \ell^{b} \nabla_{b} \ell_{a} & \epsilon=\frac{1}{2}\left(\bar{m}^{a} \ell^{b} \nabla_{b} m_{a}-n^{a} \ell^{b} \nabla_{b} \ell_{a}\right) & \pi=\bar{m}^{a} \ell^{b} \nabla_{b} n_{a} \\
\sigma=-m^{a} m^{b} \nabla_{b} \ell_{a} & \beta=\frac{1}{2}\left(\bar{m}^{a} m^{b} \nabla_{b} m_{a}-n^{a} m^{b} \nabla_{b} \ell_{a}\right) & \mu=\bar{m}^{a} m^{b} \nabla_{b} n_{a} \\
\rho=-m^{a} \bar{m}^{b} \nabla_{b} \ell_{a} & \alpha=\frac{1}{2}\left(\bar{m}^{a} \bar{m}^{b} \nabla_{b} m_{a}-n^{a} \bar{m}^{b} \nabla_{b} \ell_{a}\right) & \lambda=\bar{m}^{a} \bar{m}^{b} \nabla_{b} n_{a} \\
\tau=-m^{a} n^{b} \nabla_{b} \ell_{a} & \gamma=\frac{1}{2}\left(\bar{m}^{a} n^{b} \nabla_{b} m_{a}-n^{a} n^{b} \nabla_{b} \ell_{a}\right) & \nu=\bar{m}^{a} n^{b} \nabla_{b} n_{a} .
\end{array}
$$

It is sometimes more useful to express these definitions in terms of covariant derivatives of the basis vectors:

$$
\begin{aligned}
D \ell & =(\epsilon+\bar{\epsilon}) \ell-\bar{\kappa} m-\kappa \bar{m} & D n & =-(\epsilon+\bar{\epsilon}) n+\pi m+\overline{\pi m} \\
\Delta \ell & =(\gamma+\bar{\gamma}) \ell-\bar{\tau} m-\tau \bar{m} & \Delta n & =-(\gamma+\bar{\gamma}) n+\nu m+\overline{\nu m} \\
\delta \ell & =(\bar{\alpha}+\beta) \ell-\bar{\rho} m-\sigma \bar{m} & \delta n & =-(\bar{\alpha}+\beta) n+\mu m+\bar{\lambda} \bar{m} \\
D m & =\bar{\pi} \ell-\kappa n+(\epsilon-\bar{\epsilon}) m & \Delta m & =\bar{\nu} \ell-\tau n+(\gamma-\bar{\gamma}) m \\
\delta m & =\bar{\lambda} \ell-\sigma n+(\beta-\bar{\alpha}) m & \bar{\delta} m & =\bar{\mu} \ell-\rho n+(\alpha-\bar{\beta}) m .
\end{aligned}
$$

The ten independent components of the Weyl tensor are expressed in terms of five complex scalars $\Psi_{0}, \Psi_{1}, \Psi_{2}, \Psi_{3}$ and $\Psi_{4}$. The ten components of the Ricci tensor are defined in terms 
of four real and three complex scalars $\Phi_{00}, \Phi_{11}, \Phi_{22}, \Lambda, \Phi_{10}, \Phi_{20}$ and $\Phi_{21}$. These scalars are defined as follows:

$$
\begin{aligned}
& \Psi_{0}=C_{a b c d} \ell^{a} m^{b} \ell^{c} m^{d} \quad \Phi_{01}=\frac{1}{2} R_{a b} \ell^{a} m^{b} \quad \Phi_{10}=\frac{1}{2} R_{a b} \ell^{a} \bar{m}^{b} \\
& \Psi_{1}=C_{a b c d} \ell^{a} m^{b} \ell^{c} n^{d} \quad \Phi_{02}=\frac{1}{2} R_{a b} m^{a} m^{b} \quad \Phi_{20}=\frac{1}{2} R_{a b} \bar{m}^{a} \bar{m}^{b} \\
& \Psi_{2}=C_{a b c d} \ell^{a} m^{b} \bar{m}^{c} n^{d} \quad \Phi_{21}=\frac{1}{2} R_{a b} \bar{m}^{a} n^{b} \quad \Phi_{12}=\frac{1}{2} R_{a b} m^{a} n^{b} \\
& \Psi_{3}=C_{a b c d} \ell^{a} n^{b} \bar{m}^{c} n^{d} \quad \Phi_{00}=\frac{1}{2} R_{a b} \ell^{a} \ell^{b} \quad \Phi_{11}=\frac{1}{4} R_{a b}\left(\ell^{a} n^{b}+m^{a} \bar{m}^{b}\right) \\
& \Psi_{4}=C_{a b c d} \bar{m}^{a} n^{b} \bar{m}^{c} n^{d} \quad \Phi_{22}=\frac{1}{2} R_{a b} n^{a} n^{b} \quad \Lambda=\frac{R}{24} .
\end{aligned}
$$

The six components of the Electromagnetic-Field 2-form $\mathbf{F}_{a b}$ can be defined in terms of three complex scalars:

$$
\phi_{0}=-\ell^{a} m^{b} \mathbf{F}_{a b} \quad \phi_{1}=-\frac{1}{2}\left(\ell^{a} n^{b}-m^{a} \bar{m}^{b}\right) \mathbf{F}_{a b} \quad \phi_{2}=n^{a} \bar{m}^{b} \mathbf{F}_{a b} .
$$

The eight real Maxwell equations $d \mathbf{F}=0$ and $d^{\star} \mathbf{F}=0$ can be written as a set of four complex equations:

$$
\begin{aligned}
D \phi_{1}-\bar{\delta} \phi_{0} & =(\pi-2 \alpha) \phi_{0}+2 \rho \phi_{1}-\kappa \phi_{2} \\
D \phi_{2}-\bar{\delta} \phi_{1} & =-\lambda \phi_{0}+2 \pi \phi_{1}+(\rho-2 \epsilon) \phi_{2} \\
\Delta \phi_{0}-\delta \phi_{1} & =(2 \gamma-\mu) \phi_{0}-2 \tau \phi_{1}+\sigma \phi_{2} \\
\Delta \phi_{1}-\delta \phi_{2} & =\nu \phi_{0}-2 \mu \phi_{1}+(2 \beta-\tau) \phi_{2} .
\end{aligned}
$$

\section{Boundary Conditions}

In this section we describe the isolated horizon boundary conditions in the NewmanPenrose formalism. We will restrict ourselves to Einstein-Maxwell theory with zero cosmological constant.

In a null-tetrad adapted to the null hypersurface $\Delta$, take $\ell$ to be a null normal, $m$ and $\bar{m}$ tangent to $\Delta$ and $n$ transverse to $\Delta$. Since $\ell$ is hypersurface orthogonal and null, it is geodesic. This implies that $\kappa_{N P} \widehat{=} 0$ and $\operatorname{Im}[\rho] \widehat{=} 0$. Thus

$$
D \ell^{b}:=\ell^{a} \nabla_{a} \ell^{b} \hat{=}(\epsilon+\bar{\epsilon}) \ell^{b} .
$$

The surface gravity is therefore given by $\kappa_{(\ell)}=\epsilon+\bar{\epsilon}$ and the expansion of $\ell$ is $\theta_{(\ell)} \widehat{=} \operatorname{Re}[\rho]$.

For a non-expanding horizon $\Delta$, the conditions on $\ell$ imply $\rho \widehat{=} 0$ and the Raychaudhuri equation then implies $\sigma \widehat{=} 0$ and $\Phi_{00}=\frac{1}{2} R_{a b} \ell^{a} \ell^{b} \widehat{=} 0$. Furthermore, from ([1.16) (which is

\footnotetext{
${ }^{16}$ We will denote the NP spin coefficient $\kappa$ by $\kappa_{N P}$ to distinguish it from the surface gravity $\kappa_{(\ell)}$.
} 
a consequence of the energy condition), it follows that $\phi_{0} \widehat{=} 0$. This leads to the following conditions on the Ricci tensor at the horizon

$$
\begin{array}{lll}
\Phi_{00} \hat{=} 0 & \Phi_{01} \hat{=} 0 & \Phi_{10} \hat{=} 0 \\
\Phi_{02} \hat{=} 0 & \Phi_{20} \hat{=} 0 & \Phi_{11} \hat{=}-2 G \phi_{1} \bar{\phi}_{1} .
\end{array}
$$

The first Maxwell equation (

$$
D \phi_{1} \widehat{=} 0 \quad \text { which implies } \quad \mathrm{D} \Phi_{11} \widehat{=} 0
$$

Also, as shown in (11.8)

$$
\Psi_{0} \widehat{=} 0 \quad \text { and } \quad \Psi_{1} \widehat{=} 0 \text {. }
$$

The intrinsically defined one-form $\omega_{a}$ defined in (II.4) is given by

$$
\omega_{a}=-\kappa_{(\ell)} n_{a}+(\alpha+\bar{\beta}) m_{a}+(\bar{\alpha}+\beta) \bar{m}_{a} .
$$

It is often convenient to choose the null tetrad such that $\underset{\longleftarrow}{ }=0$ which implies

$$
\mu \widehat{=} \bar{\mu} \quad \text { and } \quad \pi \hat{=} \alpha+\bar{\beta} \text {. }
$$

In this case we get a foliation of $\Delta$ spanned by $m$ and $\bar{m}$. Furthermore, by an appropriate spin transformation, we can choose $\epsilon$ to be real so that $\epsilon \widehat{=} \bar{\epsilon}$ and thus the foliation is Lie dragged along $\ell$ :

$$
\mathcal{L}_{\ell} m^{a}=(\epsilon-\bar{\epsilon}) m^{a} \widehat{=} 0
$$

The one-form $\omega$ now becomes

$$
\omega_{a}=-\kappa_{(\ell)} n_{a}+\pi m_{a}+\bar{\pi} \bar{m}_{a}
$$

Let us consider a weakly isolated horizon $(\Delta,[\ell])$. The condition $\mathcal{L}_{\ell} \omega=0$ is equivalent to requiring

$$
\mathcal{L}_{\ell} \pi \hat{=} 0 \quad \text { and } \quad \mathcal{L}_{\ell} \kappa_{(\ell)} \widehat{=} 0
$$

and as we proved in Section [1B, these conditions imply that the surface gravity $\kappa_{(\ell)}$ is constant on $\Delta$.

As mentioned in Section IIB, a weakly isolated horizon with non-zero surface gravity admits a natural foliation. In the Newman-Penrose framework this foliation can be characterized as follows: It is the unique foliation on each leaf of which the pull-back of the 1-form $\pi m_{a}+\bar{\pi} \bar{m}_{a}$ is divergence-free. This condition was first introduced by Hájiček 433 in the context of stationary spacetimes.

Finally, since our boundary conditions require that $\mathcal{L}_{\ell} \kappa_{(\ell)} \widehat{=} 0$, in a sense, a part of the zeroth law is simply assumed. As mentioned in Section IIB, we could have used a slightly 
different set of boundary conditions which make no direct requirement on $\kappa_{(\ell)}$ and yet lead to the zeroth law (as well as the results of Sections III - VII).

Let $(\Delta,[\ell])$ be a non-expanding horizon, equipped with an equivalence class $[\ell]$ of null normals to related to each other by constant positive rescalings. As above introduce a null tetrad where $\ell$ is an element of $[\ell], m$ and $\bar{m}$ are tangent to the foliation, $n$ is curl free and $\epsilon$ is real. In place of Definition 2, let us assume that $\Delta$ admits a foliation by a family $S_{\Delta}$ of 2 -spheres transverse to $[\ell]$ such that the NP spin coefficients in an associated null-tetrad satisfy:

$$
\mathcal{L}_{\ell} \mu \widehat{=} 0 \quad \text { and } \quad \mathcal{L}_{\ell} \pi \widehat{=} 0
$$

These conditions now replace the requirement $\mathcal{L}_{\ell} \omega \hat{=} 0$ used in the definition of a weakly isolated horizon. We can prove the zeroth law from these conditions as follows. First, consider the definition $2 \nabla_{[a} \nabla_{b]} \xi_{c}=R_{a b c}{ }^{d} \xi_{d}$ of the Riemann tensor. In the NP formalism, these are written as a set of 18 complex equations known as the 'field equations'. For our purposes, we need only three of these equations 42]

$$
\begin{aligned}
D \alpha-\bar{\delta} \epsilon & =(\rho+\bar{\epsilon}-2 \epsilon) \alpha+\beta \bar{\sigma}-\bar{\beta} \epsilon-\kappa \lambda-\bar{\kappa} \gamma+(\epsilon+\rho) \pi+\Phi_{10} \\
D \beta-\delta \epsilon & =(\alpha+\pi) \sigma+(\bar{\rho}-\bar{\epsilon}) \beta-(\mu+\gamma) \kappa-(\bar{\alpha}-\bar{\pi}) \epsilon+\Psi_{1} \\
D \mu-\delta \pi & =(\bar{\rho}-\epsilon-\bar{\epsilon}) \mu+\sigma \lambda+(\bar{\pi}-\bar{\alpha}+\beta) \pi-\nu \kappa+\Psi_{2}+2 \Lambda .
\end{aligned}
$$

Adding the first equation to the complex conjugate of the second equation and imposing our boundary conditions gives

$$
\delta(\epsilon+\bar{\epsilon}) \widehat{=} \delta \kappa_{(\ell)} \widehat{=} 0
$$

while the third equation reduces to

$$
\Psi_{2} \widehat{=}(\epsilon+\bar{\epsilon}) \mu
$$

Equation (B.21) tells us that surface gravity is constant on each leaf of the foliation. It now only remains to show that it is also constant along $\ell$. To show this we turn to the Bianchi identity: $\nabla_{[a} R_{b c] d e}=0$. In the NP formalism, this is written as a set of nine complex and two real equations. We shall need only two of these equations [42]

$$
\begin{aligned}
D \Psi_{2}- & \bar{\delta} \Psi_{1}+\Delta \Phi_{00}-\bar{\delta} \Phi_{01}+2 D \Lambda \\
= & -\lambda \Psi_{0}+2(\pi-\alpha) \Psi_{1}+3 \rho \Psi_{2}-2 \kappa \Psi_{3}+\bar{\sigma} \Phi_{02} \\
& +(2 \gamma+2 \bar{\gamma}-\bar{\mu}) \Phi_{00}-2(\alpha+\bar{\tau}) \Phi_{01}-2 \tau \Phi_{10}+2 \rho \Phi_{11} \\
& \\
D \Phi_{11}- & \delta \Phi_{10}+\Delta \Phi_{00}-\bar{\delta} \Phi_{01}+3 D \Lambda \\
= & (2 \gamma+2 \bar{\gamma}-\mu-\bar{\mu}) \Phi_{00}+(\pi-2 \alpha-2 \bar{\tau}) \Phi_{01}+(\bar{\pi}-2 \bar{\alpha}-2 \tau) \Phi_{10} \\
\quad & +2(\rho+\bar{\rho}) \Phi_{11}+\bar{\sigma} \Phi_{02}+\sigma \Phi_{20}-\bar{\kappa} \Phi_{12}-\kappa \Phi_{21} .
\end{aligned}
$$


Subtracting these equations, imposing our boundary conditions and using $\Lambda=0$, we get $D \Psi_{2} \widehat{=} 0$. Combining this result with $(\widehat{\mathrm{B} .22})$ gives $D(\epsilon+\bar{\epsilon}) \widehat{=} 0$. This completes the proof of the zeroth law within the alternate definition of weak isolation. Most of the results of this paper were first obtained using that definition. However, since that notion is tied so heavily to the presence of a foliation, its intrinsic meaning is somewhat obscure. Therefore, it was then replaced by Definition 2 used in the main body of the paper. 


\section{REFERENCES}

[1] J.D. Bekenstein, Black holes and entropy, Phys. Rev. D7 2333 (1973);

J.D. Bekenstein, Generalized second law of thermodynamics in black hole physics Phys. Rev. D9 3292 (1974);

J.D. Bekenstein and A. Meisels, Einstein A and B coefficients for a black hole Phys. Rev. D15 2775 (1977).

[2] J.W. Bardeen, B. Carter and S.W. Hawking, The four laws of black hole mechanics Commun. Math. Phys. 31161 (1973).

[3] B. Carter, Black hole equilibrium states Black Holes, ed B DeWitt and C DeWitt (Gordon and Breach: New York) (1973).

[4] S.W. Hawking, Particle creation by black holes Commun. Math. Phys. 43199 (1975).

[5] M. Heusler, Black Hole Uniqueness Theorems (Cambridge University Press: Cambridge) (1996).

[6] A. Ashtekar, A. Corichi and K. Krasnov, Isolated horizons: the classical phase space, Adv. Theor. Math. Phys. 3 418-471 (1999) (Preprint gr-qc/9905089).

[7] A. Ashtekar, C. Beetle and S. Fairhurst, Isolated horizons: a generalization of black hole mechanics Class. Quantum Grav. 16 L1 (1999).

[8] A. Ashtekar, C. Beetle and S. Fairhurst, Mechanics of isolated horizons, Class. Quantum Grav. 17 253-298 (2000).

[9] A. Ashtekar and A. Corichi, Laws governing isolated horizons: inclusion of dilaton coupling, Class. Quantum Grav. 17 1317-1332 (2000).

[10] R.M. Wald, Quantum Field Theory in Curved Spacetime and Black Hole Thermodynamics (University of Chicago Press) (1994).

[11] G. Gibbons and S.W. Hawking, Cosmological event horizons, thermodynamics, and particle creation Phys. Rev. D15 2738 (1977).

[12] A. Corichi and D. Sudarsky, Mass of colored black holes Phys. Rev. D61 101501 (2000); A. Corichi, U. Nucamendi and D. Sudarsky, Einstein-Yang-Mills Isolated Horizons: Phase Space, Mechanics, Hair and Conjectures (preprint gr-qc 0002078) (2000).

[13] A. Ashtekar, C. Beetle and J. Lewandowski, Mechanics of rotating isolated horizons (in preparation) (2000); C. Beetle, Isolated horizons and black hole mechanics Penn State Ph.D. dissertation (2000).

[14] D. Brown and J.W. York, Quasilocal energy and conserved charges derived from the gravitational action Phys. Rev. D 47 1407-1419 (1993)(gr-qc/9209012);

[15] P.T. Chruściel, On the global structure of Robinson-Trautman space-time, Proc. Roy. Soc. London A 436 299-316 (1992).

[16] C. Beetle and A. Corichi (in preparation).

[17] D. Kastor and J. Traschen, Phys. Rev. D 475370 (1993);

K. Nakao, T. Shiromizu and S. Hayward, Horizons of the Kastor-Traschen multi-blackhole cosmos, Phys. Rev. D 52 796-808 (1995).

[18] A. Ashtekar, C. Beetle and J. Lewandowski, Geometry of general isolated horizons (in preparation) (2000). 
A. Ashtekar, C. Beetle, O. Dreyer, S. Fairhurst, B. Krishnan, J. Lewandowski and J. Wiśniewski, Generic Isolated Horizons and their Applications, Phys. Rev. Lett. In Press.

[19] A. Ashtekar, Radiative degrees of freedom of the gravitational field in exact general relativity J. Math. Phys. 22, 2885-2895 (1981);

A. Ashtekar and M. Streubel, Symplectic geometry of radiative modes and conserved quantities at null infinity Proc. R. Soc. (London) A376, 585-607 (1981).

[20] A. Ashtekar, J. Baez, A. Corichi and K. Krasnov, Quantum geometry and black hole entropy Phys. Rev. Lett. 80 904-907 (1998);

A. Ashtekar, J. Baez, and K. Krasnov, Quantum geometry of isolated horizons and black hole entropy, pre-print NSF-ITP-99-153 (1999).

[21] A. Ashtekar, Lectures on Non-Perturbative Canonical Gravity, Notes prepared in collaboration with R. S. Tate, (World Scientific) (1991).

[22] A. Ashtekar, L. Bombelli and O. Reula, The covariant phase space of asymptotically flat gravitational fields Mechanics, Analysis and Geometry: 200 years after Lagrange ed. M Francaviglia (North-Holland) (1991);

R.M. Wald and A. Zoupas, General Definition of "Conserved Quantities" in General Relativity and Other Theories of Gravity Phys. Rev. D61 084027 (2000).

[23] D. Sudarsky and R.M. Wald, Extrema of mass, stationarity and staticity, and solutions to the Einstein-Yang-Mills equations, Phys. Rev. 46 1453-1474 (1992).

[24] M. Heusler and N. Straumann, The first law of black hole physics for a class of non-linear matter models, Class. Quant. Grav. 10 1299-1321 (1993).

[25] A.K.M. Masood-ul-Alam, Uniqueness of a static charged dilaton black hole, Class. Quant. Grav. 10 2649-2656 (1993).

[26] G. Gibbons, R. Kallosh and B. Kolb, Moduli, scalar charges and the first law of black hole thermodynamics Phys. Rev. Lett. 77 4992-4994 (1996).

[27] B. Kleihaus and J. Kunz, Static black holes with axial symmetry Phys. Rev. Lett. 79 1595-1598 (1998);

B. Kleihaus and J. Kunz, Static axially symmetric Einstein-Yang-Mills-Dilaton solutions: 1. Regular solutions Phys. Rev. D57 834-856 (1998);

B. Kleihaus and J. Kunz, Static axially symmetric Einstein-Yang-Mills-Dilaton solutions: 2. Black hole solutions Phys. Rev. D57 6138- 6157 (1998).

[28] R. Bartnik and J. McKinnon, Particlelike solutions of the Einstein-Yang-Mills Equations Phys. Rev. Lett. 61 141-144 (1988);

P. Bizon, Colored Black Holes Phys. Rev. Lett. 64 2844-2847 (1990).

D. Brown and J.W. York, Microcanonical functional integral for the gravitational field Phys. Rev. D 47 1420-1431 (1993) (gr-qc/9209014).

[29] R.M. Wald, The thermodynamics of black holes Living Reviews in Relativity (1999).

[30] B. Julia and S. Silva, Currents and superpotentials in classical gauge theories: II. Global aspects and the example of affine gravity (preprint gr-qc/0005127) (2000).

[31] A. Ashtekar, O. Dreyer and J. Wiśniewski, Isolated horizons in $2+1$ dimensions (in preparation).

[32] R.M. Wald, Black hole entropy is Noether charge. Phys. Rev. D48 3427-31 (1993); 
V. Iyer and R.M. Wald, Some Properties of Noether charge and a proposal for dynamical black hole entropy. Phys. Rev. D50 846-64 (1994).

[33] J. Lewandowski, Space-times admitting isolated horizons Class. Quantum Grav. (in press) Preprint gr-qc/9907058.

[34] F.J. Ernst, Black holes in a magnetic universe J. Math. Phys. 17 No.1, 54-56 (1976).

[35] L. Mysak and G. Szekeres, Behavior of the Schwarzschild Singularity in Superimposed Gravitational Fields Can.J.Phys. 44617 (1966);

R. Geroch and J.B. Hartle, Distorted Black Holes J. Math. Phys. 23(4) 680 (1982).

[36] S. Fairhurst and B. Krishnan, Distorted black holes with charge (in preparation).

[37] H. Weyl, Zur Gravitationstheorie Ann. Physik 54 117-145 (1917).

[38] R. Gatreau, R.B. Hoffman and A. Armenti jr, Static Multi-Particle Systems in General Relativity Il Nuovo Cimento 7B(1) 71 (1972).

[39] E.T. Newman and R. Penrose, An approach to gravitational radiation by a method of spin coefficients J. Math. Phys 3 566-578 (1962).

[40] R. Penrose and W. Rindler, Spinors and Space-Time (Cambridge University Press: Cambridge) (1984).

[41] S. Chandrasekhar, The Mathematical Theory of Black Holes (Oxford Classic Texts in the Physical Sciences) (1998).

[42] J. Stewart, Advanced General Relativity (Cambridge Monographs on Mathematical Physics) (1991).

[43] P. Hájiček, Stationary electrovacuum spacetimes with bifurcate horizons J. Math. Phys. 16 518-522 (1975). 